\title{
Intelligent system for COVID-19 prognosis: a state-of-the-art survey
}

\author{
Janmenjoy Nayak ${ }^{1} \cdot$ Bighnaraj Naik $^{2}$ (D) Paidi Dinesh ${ }^{3} \cdot$ Kanithi Vakula $^{3} \cdot$ B. Kameswara Rao ${ }^{1} \cdot$ Weiping Ding ${ }^{4}$. \\ Danilo Pelusi ${ }^{5}$
}

Accepted: 27 November 2020 / Published online: 6 January 2021

(C) The Author(s), under exclusive licence to Springer Science+Business Media, LLC part of Springer Nature 2021

\begin{abstract}
This 21 st century is notable for experiencing so many disturbances at economic, social, cultural, and political levels in the entire world. The outbreak of novel corona virus 2019 (COVID-19) has been treated as a Public Health crisis of global Concern by the World Health Organization (WHO). Various outbreak models for COVID-19 are being utilized by researchers throughout the world to get well-versed decisions and impose significant control measures. Amid the standard methods for COVID-19 worldwide epidemic prediction, easy statistical, as well as epidemiological methods have got more consideration by researchers and authorities. One main difficulty in controlling the spreading of COVID-19 is the inadequacy and lack of medical tests for detecting as well as identifying a solution. To solve this problem, a few statistical-based advances are being enhanced and turn into a partial resolution up-to some level. To deal with the challenges of the medical field, a broad range of intelligent based methods, frameworks, and equipment have been recommended by Machine Learning (ML) and Deep Learning. As ML and DL have the ability of identifying and predicting patterns in complex large datasets, they are recognized as a suitable procedure for producing effective solutions for the diagnosis of COVID-19. In this paper, a perspective research has been conducted in the applicability of intelligent systems such as ML, DL and others in solving COVID-19 related outbreak issues. The main intention behind this study is (i) to understand the importance of intelligent approaches such as ML and DL for COVID-19 pandemic, (ii) discussing the efficiency and impact of these methods in the prognosis of COVID-19, (iii) the growth in the development of type of ML and advanced ML methods for COVID-19 prognosis,(iv) analyzing the impact of data types and the nature of data along with challenges in processing the data for COVID-19,(v) to focus on some future challenges in COVID-19 prognosis to inspire the researchers for innovating and enhancing their knowledge and research on other impacted sectors due to COVID-19.
\end{abstract}

Keywords COVID-19 $\cdot$ Machine learning $\cdot$ Deep learning $\cdot$ Mathematical model $\cdot$ Intelligent system

\section{Introduction}

Over the past 50,000 to 100,000 years, modern humans are increasing and dispersed all over the world [1]. There has been

\author{
Bighnaraj Naik \\ bnaik_mca@vssut.ac.in \\ Janmenjoy Nayak \\ jnayak.cse@adityatekkali.edu.in \\ Paidi Dinesh \\ dinesh.pydi98@gmail.com \\ Kanithi Vakula \\ vakku.bi@gmail.com \\ B. Kameswara Rao \\ kamesh3410@gmail.com \\ Weiping Ding \\ dwp9988@163.com
}

rehabilitated official and community apprehension about the contagious disease as a foremost public health hazard. Certainly, the concern has appeared against a backdrop of various surprises. After all, we survive in hygienic homes with

Danilo Pelusi
dpelusi@unite.it

1 Department of Computer Science and Engineering, Aditya Institute of Technology and Management (AITAM), K Kotturu, Tekkali, AP 532201, India

2 Department of Computer Application, Veer Surendra Sai University of Technology, Burla, Odisha 768018, India

3 Department of Computer Science and Engineering, Sri Sivani College of Engineering, Srikakulam, AP 532402, India

4 School of Information Science and Technology, Nantong University, Nantong, China

5 Faculty of Communication Sciences, University of Teramo, Coste Sant\&\#39, Agostino Campus, Teramo, Italy 
scented toilet bowls, confined by a surplus of antibiotics and immunizations and consume pressure-packed uncontaminated foods. Definitely, sterilized fantasy is near. But, modern incidents indicate differently. In the earlier century, novel contagious diseases appeared including those which were originated through viruses. We have detected the appearance of Legionnaire's disease, the Nipah virus, HIV/AIDS, Lyme disease, West Nile fever, human mad cow disease, severe acute respiratory syndrome SARS, the Middle East respiratory syndrome (MERS), measles, hepatitis virus, and Ebola virus, as well as revived challengers such as smallpox, malaria, dengue fever, tuberculosis, and cholera. It shows that contagious diseases are rising, falling, and dispersing more liberally in the current world. According to world health organization (WHO), more than 30 novel diseases like SARS corona virus (SARS-COV), MERS corona virus (MERS-COV), and H1N1 influenza, etc., have occurred in the past 20 years to intimidate the physical condition of millions of people. Long-distance trade and human flexibility have improved, huge cities, frequently encircled with slums have developed into a freeway for microbial traffic; sexual practices, drug inserting, severed food production, Poverty effects susceptibility to contagious disease and much recent medical tools all produce innovative prospects for microbial opportunism. In the current century, worldwide pandemic with distress deaths has been caused by SARS-COV and MERS-COV which are two extremely pathogenic individual corona viruses that have initiated from the animals.

Corona viruses are a set of associated viruses that cause illness in birds and mammals. The name was derived from Latin corona which means 'crown' and name itself refers to the feature appearance of the club-shaped and crown-like spikes on the external surface of the virus. Corona viruses are huge, encircled, positive-strand RNA viruses. Corona virus $(\mathrm{COV})$ belongs to the sub-family of Ortho corona virinae and part of a corona viridae family. Corona viruses divided into four genera's such as alpha, beta, gamma, and delta COV's, where mammals were infected through alpha and beta COV's and birds were infected through gamma and delta COV's respectively [2]. COV was first identified in the late 1930s when a severe respiratory virus of domestic chickens was exposed to be affected by the infectious bronchitis virus (IBV) [3]. Later in 1960, human corona viruses (HCOVs) were recognized. COV in humans can be caused by respiratory tract diseases which vary from mild to lethal. Where mild infection consists of few cases of general cold and lethal infection consists of SARS as well as MERS. Both these SARS and MERS belong to Beta COV's, which is a brutal and malignant respiratory disease.

Another pathogenic HCOV was out broke in December 2019 and was recognized in Wuhan, China [4] and traced as a new injure of corona virus. The city of Wuhan is the main transport center with more than 11 million populations [5]. Wuhan city is famous for selling various live animals such as bats, snakes, marmots, poultry, etc. and most of the patients were found visiting that wild animal market before December. A total of 44 cases with pneumonia of unidentified etiology were reported in Wuhan from 31 to 2019 to 3 January 2020. The reason behind the virus was not identified during the informed period. But, on 7 January 2020, WHO and the government of China have identified the new corona virus (Ncov2019) which belongs to the SARS virus family [6]. Ncov2019 was increased rapidly in the major areas of China after 17 January 2020 and traversed more than 7000 cases. Due to this, the Chinese government has taken strong measures to prohibit the transmission of this dangerous virus from 23 to 2020 by temporarily delaying public transportation, alerting citizens from leaving outside, and taking isolation for the major infected cities. On 30 January 2020, WHO acknowledged the epidemic as the novel COVID-19 extends to 18 countries by human-to-human transmission. According to the information from Worldometer.com, as many as 32,118,316 people worldwide are affected by COVID-19 as on September 24th 2020, and WHO has declared it a pandemic. The USA has the most confirmed cases with 7,139,553 people in the world infected with this pandemic. The death rate of the USA has stood higher with 206,593 lives. Sudden increment of severe cases and death rates in the USA as well as India gave a big shock to the entire world. Likewise, many other countries like India, Brazil, Russia, Peru, Colombia, Mexico, Africa, Spain, and Argentina are placed as a top 10 countries that are affected dangerously due to COVID-19, and their death rates, as well as recovery rates, are mention in Fig. 1 respectively.

Similarly in the case of India, the active cases have been reached to 5,732,518 and 4,674,987 patients are discharged and the death rate has been reached to 91,173 by 24 th September, 2020. The statistics of death rates (DR), recovery rates (RR) as well as positive rates (PR) of India are mentioned in Fig. 2.

The major reason behind the spreading of COVID-19 is not attaining proper information regarding early phase symptoms. This has lead to a state where citizens are unknown that they are contaminated and traveled with no knowledge about the carrying of disease. So, widespread COVID-19 made necessitate managing crowded places like bus stops, airports, and railway stations respectively. It needs many scrutinizing equipment united with thermal sensors, Machine Learning (ML) [7], and Artificial Intelligence (AI) [8]. One of the standard techniques of diagnosing COVID-19 was Reverse Transcription Polymerase Chain Reaction (RT-PCR) [9]. Short sensitivity, as well as time, cannot convene the standard of identifying quickly raising positive cases and it became one of the major disadvantages of RT-PCR. To alternate this challenge, computer tomography and $\mathrm{x}$-ray images can offer fast screening to detect the problem. Due to the complexity of the problem in the improvement of epidemiological methods, ML has newly got concentration and has a rapid growth in resolving various problems like speech and object identification, 
Fig. 1 List of statistics of death, recovery $\&$ active cases of top 10 countries in the world

\section{Death, Recovery\& Active Covid-19 Cases in top effected countries throughout the world}



image classification, etc. It is noteworthy to mention that, unlike other problem domains, the experiments on COVID19 with ML have immensely increased within a span of just two months. This indicates the importance of realizing the perilous nature of this disease and need of high level research with apposite intelligent computing approaches.

From the past decade, the fast growth of digital methodologies is playing a vital role in resolving different problems of the health sector as well as disease prevention. The existing global health emergency is also tracking the support of digital equipment to deal with COVID-19 proficiently. The prophecy of the patient's outcome is still a tough concern in the field of health care. Due to the huge growth of ML techniques, predicting and diagnosing diseases made it easy in current days. ML can learn through machines and makes a proper prediction by self-learning. ML has extensively forecasted many diseases like hepatitis [10], Human immunodeficiency virus (HIV) [11], Ebola virus [12], etc. Similarly, huge ranged data of COVID-19 patients have also been combined and studied with ML. Several techniques of ML were utilized to identify the affected individuals with personalized characteristics. The performance of ML is reliant on choosing data illustration. Although ML has shown its effectiveness at identifying a disease, gathering information, organizing of health records, etc., but a few systematic models on ML has not used yet, because of numerous confronts such as lack of identifying tools, less medical tools, utilization of biomedical data, heterogeneity, etc. To stabilize these challenges, feature engineering advanced by taking the advantage of human creativity. Yet, these drawbacks have been elaborated by some health ideologies that are consumed in the generalization of information. There can be a situation where some medical works need to be implied in unusual ways. This problem can be resolved by illustrating the health composition in the adhoc technique. Still, the latest prototypes cannot be predicted by supervised models. Nevertheless, representation learning can be effortless to extract essential data while creating predictors [13]. Among the diverse methods of representation learning, DL methods are easy and created by the composition of numerous nonlinear variations.
Fig. 2 Death, recovery \& active cases of COVID-19 in India

\section{Death, Recovery\& Active Covid-19 Cases in India}




DL is a rapidly becoming significant intelligent computing method in image or video categorization and identification. DL has been used in several medical applications such as disease diagnosis, drug design, medical image analysis etc. The architecture of DL can also be utilized in many areas of image processing as well as computer vision that includes the ability to hold complex data and constant learning systems with shared qualities. DL enhances a health system to recognize superior outcomes, executing relevant immediate medical images, and expand the reduction chances of infection scope [14]. In recent years, DL has been used to recognize infection in the human body automatically [9]. The prediction of COVID-19 with DL plays a crucial role and it has been a recent interest for detection and prediction of this pandemic. Moreover, identification of tumor classification, nodule classification, prostate segmentation, etc. has been effectively experimented through DL techniques. DL is the most efficient supervised ML advance [15]. Patients with a similar analysis may respond diversely by their health, genetic prompting, and demographics. DL has outperformed various researches with dependable information on improving the effectiveness of the medical trials by forecasting creation ways and side effects. As everything provides possibilities, it becomes obvious that clinicians require identifying when the correct time to use ML or DL. The major characteristics such as better performance, lack of human involvement in quality extraction and recognition made the DL techniques as more efficient and popular way over ML techniques for prediction of COVID-19. In this way, both ML and DL have showcased their efficiencies and differentiate themselves in different ways especially in health care. ML is an exclusive technology that is used to allow a computer to train itself and make correct decisions and can be employed for predicting coupled information from previous records, geography, and demographics, climate as well as outbreak severity. Image acquisition machines have enhanced significantly and presently we are receiving radiological images with much-advanced resolution. Conventional learning techniques are not consistent due to the wide distinction data from one patient to another. ML has developed over the previous years by its capability to change through difficult and large data. On the other hand, DL can be used to practice several layers of nonlinear data and take exact decisions for a difficult problem like a result of clinical tracks.

The objective of this study is to differentiate the methodological significance among various intelligent based models such as ML as well as DL models for COVID-19 pandemic and to discuss the efficiency and impact of these methods in the prognosis of COVID-19. Many important factors such as growth in the development of ML and DL methods for various problems of COVID-19, publication advances of both methods for COVID-19, advantages of both approaches over other mathematical and statistical approaches etc. are critically analyzed with a further discussion on the challenges of developing intelligent methods. Moreover, the importance in the usage of clinical data and internet data in the prognosis of COVID-19 are thoroughly discussed. This paper has been segmented in the following manner. Section 2 describes the systematic literature review process conducted in this paper. Section 3 illustrates the usage levels of ML for COVID-19 along with its applications. Detailed analysis on DL for COVID-19 along with its applications is mentioned in Section 4. Section 5 describes the types of data and its nature used for resolving COVID-19 related conflicts. A thorough critical analysis with all methodological advances along with the impact of intelligent methods in COVID-19 has been mentioned in Section 6. Various sectors affected due to COVID19 outbreak are mentioned in Secttion 7. Some of the discussions with current and future stage of research analyzed from the study are mentioned in Section 8 . Section 9 concludes this paper with some important future directions.

\section{Systematic literature review process}

Intelligent systems such as machine learning, deep learning etc. have been used almost everywhere in the world. Dayby-day many researches with the help of these intelligent systems are being made and several articles are being published in reputed online databases. These intelligent systems especially ML and DL are being applied in various applications such as cyber physical systems, IoT, healthcare etc. After recognizing the pandemic COVID19 outbreak in Wuhan, China in the month of December, 2019, the significance of these intelligent systems has been drastically increased in solving the problems of COVID19. Since from its recognition, many researches are being initiated day-by-day in an increasing fashion. Taking these factors as inspiration, in this paper, an analysis has been made on the usage levels of ML as well as DL techniques for solving COVID-19 techniques. For this, we have used a special article search strategy for paper collection. Keywords "Machine Learning for COVID-19"; "Deep Learning for COVID-19" has been applied on several benchmark databases such as Google Scholar, IEEE Xplore, Elsevier, Springer, etc. The paper search strategy has been depicted in Fig. 3. On the basis of keyword search, many papers are found related to COVID-19. As our focuses are on ML as well as DL techniques, we have only considered papers related to ML \& DL. We have used inclusion as well as exclusion procedures for filtering papers.

Inclusion Procedures for filtering papers related to COVID19 are considered as follows:

1. Considering papers related to ML methods applied for solving COVID-19 issues. 
Fig. 3 Paper search procedure considered for the study

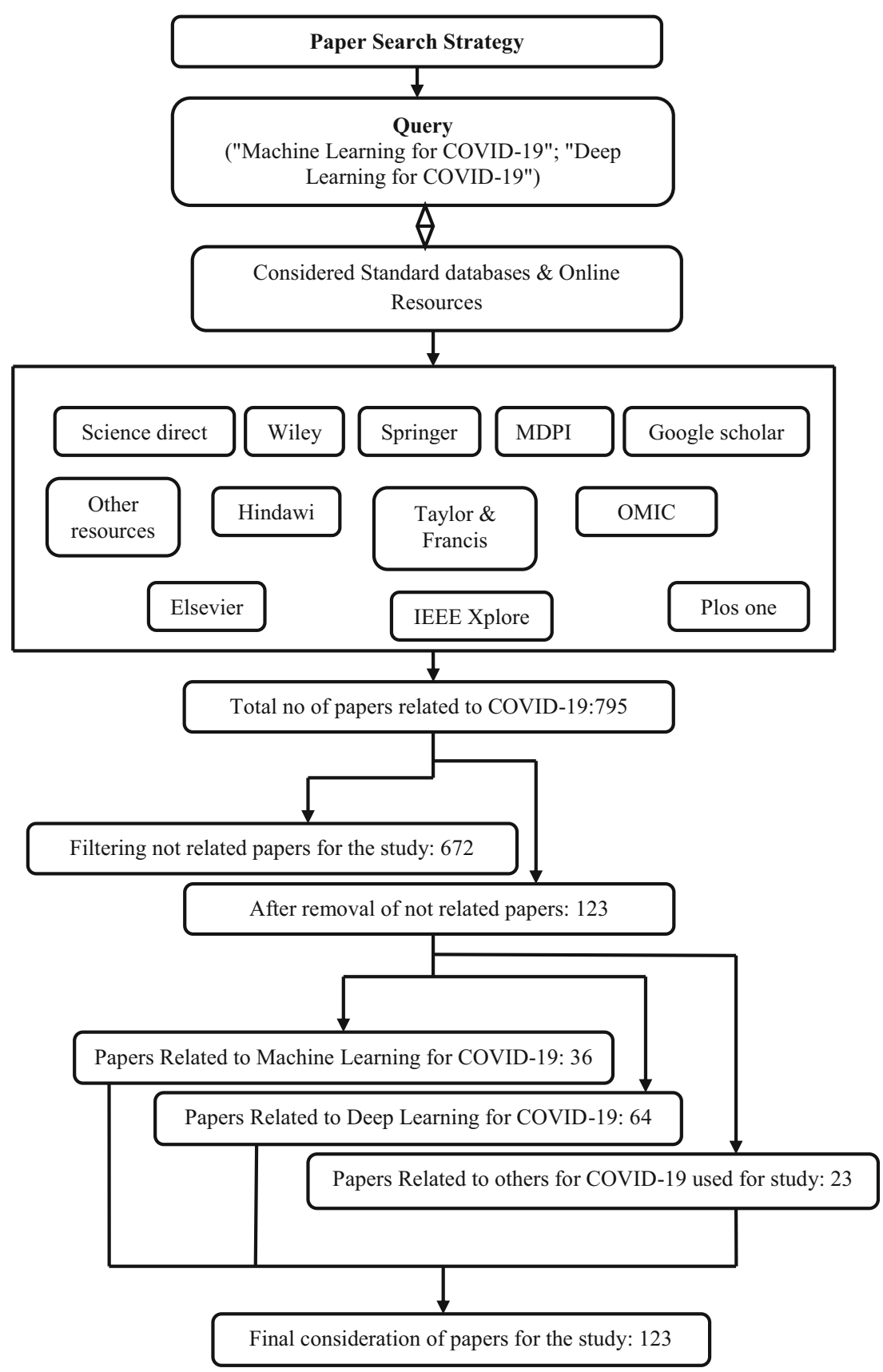

2. Considering papers related to DL methods for solving COVID-19 problems.

3. Considering papers related to COVID-19 news, datasets, practice guidelines, biological processes and others.

Also, exclusion Procedures for filtering papers related to COVID-19 are considered as follows:

1. Papers or abstracts related to COVID-19 but not accessible.

2. Papers related to COVID-19 published in case reports, product reviews, video articles etc.
3. Papers that are not related to COVID-19.

After applying these inclusion as well as exclusion procedures, all papers that are essential to use in this study are considered. A total number of 123 papers have been considered for the final study. Out of those 123 papers, 64 papers are DL related, 36 are ML related and rest of 23 is related to others. These 123 papers are extracted from various databases such as science direct, Wiley, Springer, MDPI, Google Scholar, Elsevier, IEEE explorer, Hindawi, Taylor and Francis, OMIC, Plos one, and some other resources. The data extracted from every single paper considered for the study are 
dataset used, method used, type of input used, type of image used, predicted outcomes as well as usage levels etc.

\section{Machine learning for COVID-19}

ML can learn through machines and concern algorithms for self-learning and making predictions. ML techniques are used to identify the affected people potentially based on physiological and personalized characteristics. Moreover, in this section, the applicability of ML for predicting, screening, detecting novel COVID-19 related researches have been summarized.

Randhawa et al. [16] has developed a machine learning (ML) approach for the identification of genomes of COVID19. The authors have proposed a decision tree approach and for the evaluation of performance, the publicly available COVID19 data with Sarbecovirus, as well as Betacorona virus is considered. Classification accuracy, prediction accuracy, etc. are considered as evaluation metrics, and based on experimental investigations; an accuracy of $100 \%$ has been obtained with the proposed model. Apostolopoulos and Mpesiana [17] have developed a modern ML approach for the detection of COVID-19 with the help of advanced ML approach named Convolutional Neural Networks (CNNs). For performance evaluation of the proposed ML model, X-ray Image [18-20] dataset has been considered and accuracy, sensitivity as well as specificity are considered as performance factors. Later, experimental investigations have been performed and a good detection rate has been observed with the developed CNN architecture. Kang et al. [21] has introduced a new automatic analysis pipeline for COVID19 that can completely influence various kinds of extracted features from CT images. A structured latent image was trained by the authors that can encode the data from various feature aspects to explore them by recounting the images from the different analyses. The authors have used KNN as well as NB methods to afford corresponding data for the COVID-19 analysis. The projected model can be broadly utilized for various classifiers to endorse the accuracy. The authors have attained a 95\% accuracy rate, $96.6 \%$ of specificity, and $93.2 \%$ of sensitivity. Cheng et al. [22] has introduced an ML-based technique that forecasts the ICU transfer for the COVID patients. The authors have utilized an RF model along with nursing assessments, laboratory information, electrocardiograms, and times series as input types. The proposed model has proved the significance of inflammation, shock, renal, and respiratory failure in COVID-19 advancement. It was found to have $72.8 \%$ sensitivity, $76.3 \%$ specificity, $79.9 \%$ AUC, and $76.2 \%$ accuracy respectively. Based on the experiments, the authors have concluded that the ML-based forecasting technique can be utilized as a testing tool to recognize the threat of COVID-19 patients and to enhance the resources of hospitals by transporting more efficient care. Khanday et al. [23] has introduced a method for identifying pandemic with clinical text information. The authors have categorized textual clinical data into 4 different classes by utilizing ensemble as well as traditional ML algorithms. The authors have used 212 clinical records that are marked in four various classes such as SARS, ARDS, COVID, and both COVID + ARDS respectively. They have performed feature engineering like a bag of words (BOW), report length, and Term frequency or inverse document frequency (TF or IDF) that were provided to the ensemble as well as conventional ML classifiers. Based on the experimentation, the author has concluded that Multinomial Naive Bayes and Logistic regression has outperformed better results with $94 \%, 96 \%, 95 \%$, and $96.2 \%$ of precision, recall, flscore, and accuracy rate. Assaf et al. [24] has used ML techniques to predict exactly the risk factors of COVID-19. The demonstration review has been made by using various medical center records of COVID-19 patients. Based on low arterial oxygen as well as oxygen saturation, the authors have excluded severe covid19 patients at the admission level only. They used three various ML techniques for predicting patient descent and contrasted to APACHE-II risk calculation score as well as presently advised predictors respectively. They assessed $6995 \mathrm{pa}-$ tients and found 162 were admitted in the hospital, and 25 were depreciated to severe covid19. Based on experimental results, the authors have concluded that the projected models have outperformed $92.0 \%, 88.0 \%$, and $92.7 \%$ rates for accuracy, sensitivity, and specificity respectively. Moreover, some other applications of ML for resolving some issues of COVID-19 have been depicted in Table 1.

\section{Deep learning for COVID-19}

DL prototype presents new aspects in the biomedical fields due to the extensive progress of practical enhancements. Ability to handle complex data, continuous learning methods, and excellent performance with shared feature learning are the characteristics of DL models that made them best appropriate for the field of healthcare. The classification technique for COVID-19 was introduced by Singh et al. [50] to categorize the contaminated patients from CT images. A new DL model was anticipated with CNN as well as multi-objective differential evolution (MODE). The authors have decomposed the CT dataset as testing and training groups. The training dataset was used for creating the COVID-19 disease categorization technique. The proposed model has been used in the training group. The authors have contrasted the projected classification techniques with competitive methods like ANN, CNN, and ANFIS by considering numerous divisions of testing and training dataset. It was found to have $1.9789 \%, 2.0928 \%$, $1.8262 \%, 1.6827 \%$, and $1.9276 \%$ of accuracy, F-measure, sensitivity, specificity, and Kappa statistics respectively. Khan et al. [51] has proposed a DL advance to identify COVID-19 from radiography images. The authors have used 
Table 1 Applications of ML for resolving some COVID-19 issues

\begin{tabular}{|c|c|c|c|c|}
\hline Dataset used & Method used & Input Type & Outcomes & Reference \\
\hline COVID-19 Time Series dataset & $\begin{array}{l}\text { LR,LASSO, Support Vector Machine } \\
\text { (SVM), ER }\end{array}$ & Text data & $\begin{array}{l}\text { ES outperforms other } \\
\text { proposed methods. }\end{array}$ & {$[25]$} \\
\hline CT Image dataset & Residual Neural Network & Image data & $91 \%$ accuracy. & {$[26]$} \\
\hline COVID-19 data of various countries & Support Vector Regression (SVR) & Text data & $\begin{array}{l}\text { It evident the necessitate for } \\
\text { carefulness while using ML. }\end{array}$ & {$[27]$} \\
\hline COVID data of 5 countries & MLP, ANFIS & $\begin{array}{l}\text { Time-series } \\
\text { data }\end{array}$ & High generalization & {$[28]$} \\
\hline $\begin{array}{l}\text { COVID-19 dataset of } 1,182 \\
\text { hospitalized patients }\end{array}$ & SVM & Text data & $\begin{array}{l}\text { Significant results has been } \\
\text { achieved in predicting recovery time. }\end{array}$ & {$[29]$} \\
\hline $\begin{array}{l}\text { COVID-19 patients data of } \\
\text { Massachusetts, Georgia, } \\
\text { and New Jersey. }\end{array}$ & GB (Gradient Boosting) algorithm & Text data & Better prediction rate & {$[30]$} \\
\hline COVID-19 Patient Dataset & ML algorithm & Text data & Good prediction rate & {$[31]$} \\
\hline COVID-19 Indian Dataset & Support vector Kuhntucker model & Text data & Better Prediction rate & {$[32]$} \\
\hline COVID-19 data from Mindstream-ai & ANN & Text data & $\begin{array}{l}\text { Better prediction rate to identify } \\
\text { infection is obtained. }\end{array}$ & {$[33]$} \\
\hline COVID-19 Data & Logistic Model + Prophet method & $\begin{array}{l}\text { Time-series } \\
\text { data }\end{array}$ & Good prediction rate & {$[34]$} \\
\hline CT dataset & $\begin{array}{l}\text { AD3D-MIL algorithm (A Deep } \\
\text { 3D-Multiple } \\
\text { Instance Learning) }\end{array}$ & Image data & An accuracy of $97.9 \%$ is obtained & {$[35]$} \\
\hline JHU CSSE database & - & Text data & Good identification rate & {$[36]$} \\
\hline Two COVID-19 chest X-ray datasets & $\begin{array}{l}\text { KNN (K Nearest Neighbor) + } \\
\text { Manta-Ray Foraging } \\
\text { Optimization (MRFO) }\end{array}$ & Image data & $\begin{array}{l}96.09 \% \text { and } 98.09 \% \text { accuracies is } \\
\text { obtained for two datasets respectively }\end{array}$ & {$[37]$} \\
\hline COVID-19 patient data & $\begin{array}{l}\text { XGB (Extreme Gradient Boosting), } \\
\text { Decision Tree (DT), Random Forest } \\
\text { (RF), SVM, GBM (Gradient } \\
\text { Boosting Machine) }\end{array}$ & Text data & $\begin{array}{l}\text { XGB outperformed other } \\
\text { proposed methods }\end{array}$ & {$[38]$} \\
\hline COVID-19 time series dataset & $\begin{array}{l}\text { Ensemble Empirical Mode } \\
\text { Decomposition (EEMD) + ANN) }\end{array}$ & Text data & Better Prediction rate & {$[40]$} \\
\hline CT images dataset & $\mathrm{CNN}, \mathrm{RF}, \mathrm{NB}, \mathrm{SVM}$, as well as JRIP & Image data & $\begin{array}{l}\text { Proposed CHFS outperformed } \\
\text { better prediction rate than } \mathrm{CNN}\end{array}$ & {$[41]$} \\
\hline COVID_CT dataset & Enhanced KNN & Image data & Good detection rate & {$[42]$} \\
\hline COVID-19 pandemic data & NN (Neural Network) & Text data & Good identification rate & {$[43]$} \\
\hline Corona virus dataset & $\begin{array}{l}\text { LR, Naive Bayes (NB), Linear } \\
\text { Regression (LiR), KNN }\end{array}$ & Text data & NB outperformed other techniques & {$[44]$} \\
\hline $\begin{array}{l}\text { Hungary dataset of } \\
\text { COVID-19 data }\end{array}$ & $\begin{array}{l}\text { ANFIS (Adaptive } \\
\text { Network-based Fuzzy Inference } \\
\text { System) } \\
\text { \& MLP-ICA (Multi Layered } \\
\text { Perceptron-Imperialist Competitive } \\
\text { Algorithm) }\end{array}$ & Text data & Good prediction rate & {$[45]$} \\
\hline COVID-19 patients data & $k$-Means algorithm & Text data & Better classification rate & {$[46]$} \\
\hline COVID-19 patients data & Support Vector Regression (SVR), RF & Text data & High prediction rate & {$[47]$} \\
\hline COVID-19 patient blood sample data & KNN, LR, RF, SVM & Text data & Better severity detection & {$[48]$} \\
\hline COVID-19 Synthetic dataset & SVR & Text data & $\begin{array}{l}\text { SVR Outperformed LiR, } \\
\text { Polynomial Regression (PLR) }\end{array}$ & [49] \\
\hline
\end{tabular}

CNN based CoroNet method to detect COVID-19 cases. They have tested and trained the proposed model on a dataset having a smaller set of images by attaining X-ray images of COVID-19 as well as several pneumonia cases from various freely accessible databases. The projected technique relied on
Xception architecture. Based on the experimental analysis, the proposed methods have attained $89.6 \%, 93 \%$, and $98.2 \%$ of accuracy, precision as well as recall rate respectively. The authors have concluded that CoroNet outperformed better outcomes and the projected technique has significantly 
progressed the existing radiology based method. Sethy and Behera [52] have developed a modern strategy for detecting the COVID-19 disease with the help of deep characteristics. To obtain deep characteristics, the authors have proposed CNNs and later these characteristics are used with the help of support vector machine (SVM). For evaluation purposes, data has been considered from GitHub, Kaggle, as well as Open-i X-ray image open-source databases and accuracy, F1 score, Kappa, etc., are considered performance factors. The proposed architecture yields better performance for detecting COVID-19 with an accuracy of 95.38 Alakus and Turkoglu [53] has proposed a new capsule network to analyze the COVID-19 using X-ray images. CNN based LSTM, CNNRNN methods were used by the authors. The proposed method was tested with data of 600 patients and confirmed with 10 -fold cross-validation approaches. The authors have utilized six various DL based applications on patients' laboratory reports. The performance metrics considered are accuracy, AUC, precision, F1-scores, as well as recall rate. It was noticed that the analytical models learned on patient's laboratory reports could be utilized to forecast infection of COVID-19. Based on the experimental results, the authors have concluded that the proposed model has outperformed $86.66 \%, 91.89 \%, 86.75 \%, 99.42 \%$, and $62.50 \%$ for accuracy, F1-score, precision, recall, and AUC respectively. Drug repurposing hopefully provides a way to develop avoidance and cure policies for COVID-19. Zeng et al. [54] has developed a comprehensive study for the identification of drugs for COVID-19 in the year 2020. A DL based model has been designed with the help of active AWS(Amazon Web Services) and 41 drug types data has been validated. TPR (True Positive Rate), FPR (False Positive Rate) etc. have been used as performance metrics and the author's proposed method is evident that DL acts as an efficient tool for discovering therapeutics. Yoo et al. [55] has developed three DL based DT classifiers for the diagnosis of COVID-19. For analysis, the dataset of chest $\mathrm{X}$-ray images has been considered. The proposed DT classifiers have been trained with CNN and accuracy, sensitivity, specificity, precision etc. have been considered as evaluation metrics. Accuracies of $98 \%, 80 \%, 95 \%$ are obtained for first, second, and third DT classifiers respectively. A good detection rate has been obtained with the proposed classifier for detecting COVID-19. Sedik et al. [56] has proposed DL models for the detection of COVID-19 in the year 2020. The two DL models considered are CNN and ConvLSTM (Convolutional Long Short-Term Memory). Datasets having chest as well as CT images are considered and accuracy, loss rate are considered as evaluation metrics. Based on the experimentations, it is observed that COVID-19 detection rate is increased with the help of proposed methodologies. Nour et al. [57] has developed a DL model for the detection of COVID-19 infection, where CNN is used as a feature extractor. Covid-19 data of chest $\mathrm{x}$-ray images is considered for performance evaluation. The deep feature extracted with help of CNN is used to feed ML techniques such as SVM, KNN, and DT. Accuracy, F-score etc. is used as performance factors. SVM yields better accuracy among other proposed methods. Jamshidi et al. [58] has developed a modern method for the diagnosis of COVID-19 with the help of intelligent DL approaches. DL approaches such as GANs, ELM (Extreme Learning Machine), LSTM are used for the study. Dataset having COVID-19 patient data (both image \& text data) is considered and evaluated using proposed methodologies. Experimentations proved that the proposed architectures act as reliable tools for diagnosing COVID-19. Waheed et al., [59] has developed a novel approach for the detection of COVID-19. The authors have addressed the problem of lack of CXR image for their research and hence proposed auxiliary GAN to produce synthetic CXR images. Later, they have used the images produced by GAN to improve the progress of $\mathrm{CNN}$ for detection of COVID-19. For the generation of dataset, publicly available datasets named IEEE CCXR (COVID Chest X-ray) dataset [60], CRD (COVID-19 Radiography Database) [61] as well as CCXR (COVID-19 Chest Xray) [62] have been considered. In order to detect COVID19 , a variant of CNN named VGG16 has been utilized and they called their whole network architecture as CGAN (COVIDGAN). Accuracy, precision as well as recall rate etc. have been considered as evaluation metrics. For detecting COVID-19 with the help of actual data, an accuracy of $85 \%$ has been obtained. After generating synthetic images with the help of GAN and utilizing it, an enhanced accuracy of $95 \%$ has been obtained for the detection of COVID-19. Chimmula et al., [63] has developed a modern method for forecasting the of COVID19 cases in Canada. Datasets offered by JHU (John Hopkins university) have been utilized for the study. For testing the nature of data considered, ADF (Augmented Dickey Fuller) test has been performed. LSTM has been used as method to forecast COVID-19 data through the adjustment of weights as selflooped gates. On Canadian data, an accuracy of $93 \%$ has been obtained for forecasting future infections of COVID-19. The authors stated that according to the experimental results obtained with LSTM model, COVID-19 will outbreak in Canada at the end of June 2020. Likewise, many research works are being performed and Table 2 evident the usage levels of DL in solving some of the conflicts of COVID-19.

\section{Critical aspects on the type of data used for COVID-19 research}

Prediction plays a major role in the epidemic and how it is out breaking. With no prediction on who is affected by the virus, 


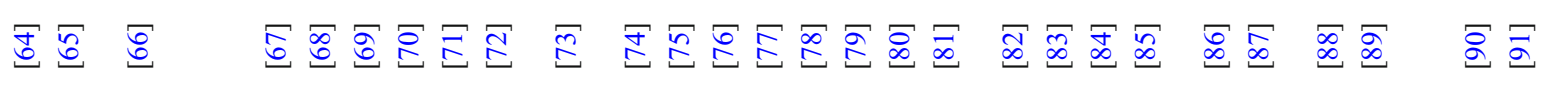

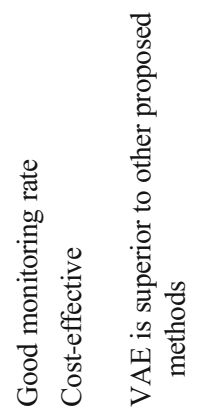



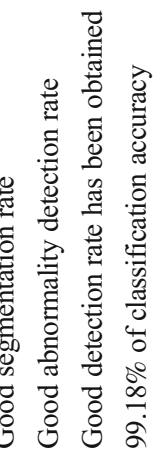<smiles>C1C[C@H]2C[C@H]1C2</smiles>
\&
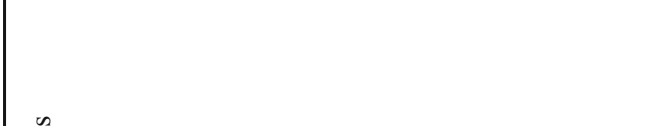

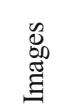<smiles>C=CCC(C)C</smiles>

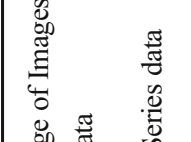<smiles>[CH][CH]</smiles>

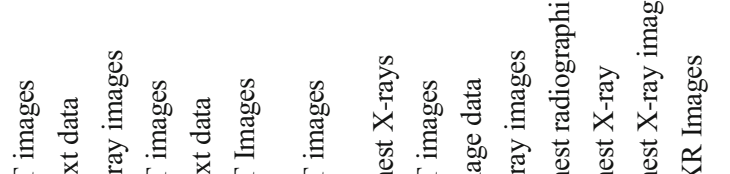

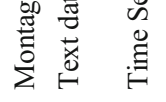

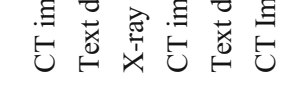

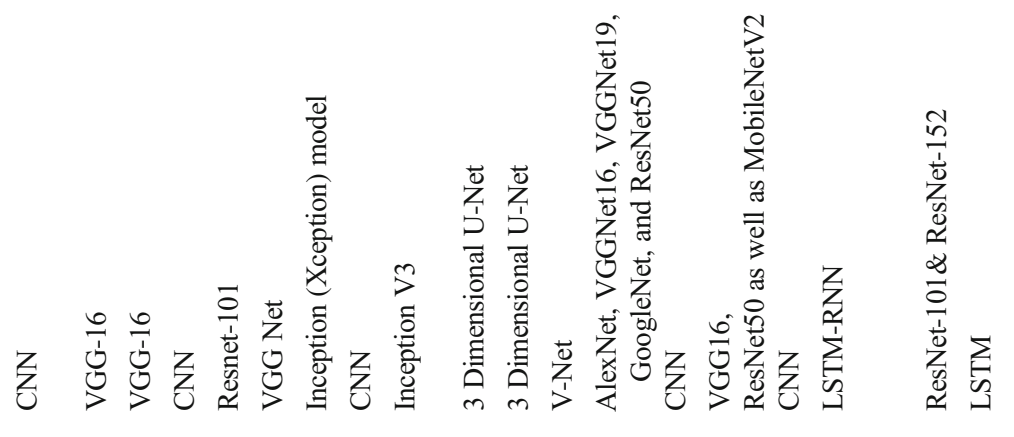




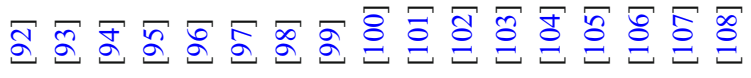

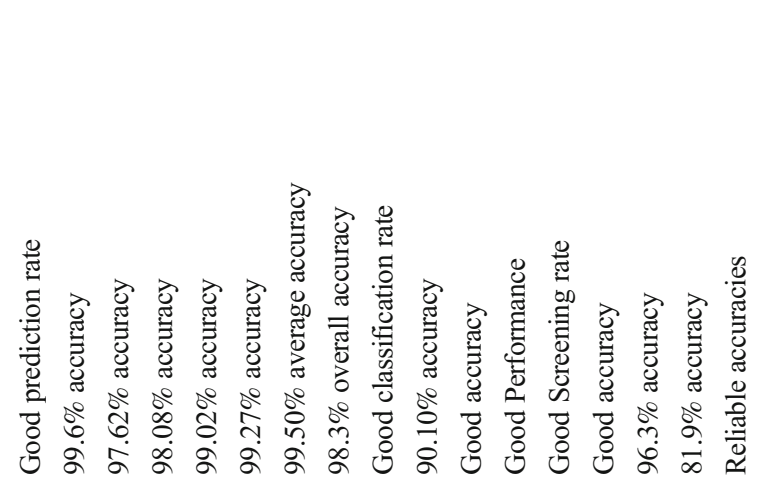

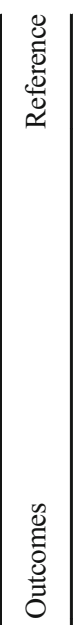

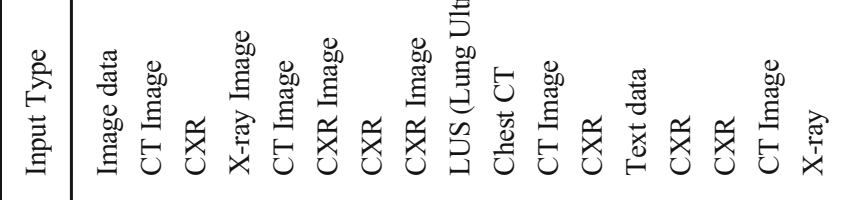


we have no way of understanding the deadly disease. Without this information, we cannot predict which countries are healthy and which are just understating the cases as well as deaths. So to predict and detect the COVID-19, researchers and doctors are using data from various domains such as online data, clinical data, and biomedical data. The following are the various kinds of data that have been utilized in the prediction of COVID-19 by the doctors as well as researchers.

\subsection{Types of data used for COVID-19}

In this subsection various types of data that have been already used for COVID-19 related researches have been described.

\subsubsection{Clinical data}

We can examine lots of data across different divisions of the world to recognize outbreaks and use higher ML as well as DL models to predict future movement across the geographies. One of the keys to gather good quality of healthcare information is from the clinical data for quick diagnostics. The prediction of the virus can be done with the number of recorded COVID-19 cases data. Mostly used datasets for the prediction of COVID-19 are Kaggle [19] dataset, Github as well as John Hopkins University [109] dataset respectively. John Hopkins University data set includes the data about PR, RR, and DR of the whole country and the Kaggle data set includes several attributes such as case reporting location, date, and patient demographics. We require finding ways to regulate various sources of data. Managing analytical abilities and datasharing formats between the public sector as well as hospitals are not simple if the country in query has a centralized patient record scheme. When functioning with existent datasets, several class disparity concerns are facing by most of the researchers. Many countries have been capable to associate health records on the nationwide health insurance database with migration and custom records to detect and test people who had newly sought healthcare, traveled from the infected areas, and demonstrated signs of brutal respiratory illness.
Still, total information standards have yet to be implemented and this produced gaps in dataset and holdups in how information can be utilized to shape overall health efforts. Another challenge that has a main impact on the prediction is the variations in involvements, demographics, and population densities. Clinical data can give accurate results for the prediction as well as analysis. So, most of the researchers have recommended clinical data under the direction of doctors for further analysis of COVID-19 when compared to other online and biomedical data and the advantages as well as disadvantage of clinical data has been depicted in below Fig. 4 .

\subsubsection{Online data}

In the current online era, most of the information is been available online that include nature, spread as well as raise of COVID-19. Usage of AI to precede a large amount of information already accessible online from travel records, community health organizations, and population records are another solution of predicting the virus. Automatic disease scrutinizing platforms are already allowing us to follow and identify the extent of disease worldwide through a combination of ML as well as DL. This process is capable to notify the outbreak of COVID-19 faster than WHO for disease handling and avoidance. Linking travel and clinical data with personal information collected from social sites, like family records and routine habits, would make it feasible to distribute even additional comprehensive predictions related to individual threat profiles and healthcare results. Daily, a huge number of online web sites and social media created a massive amount of information in various fields like medicine, technology, political, history, social news, arts, etc. The development of the pandemic is quite difficult as the majority of investigations have considered some factors. Supposing the influence of pandemic in terms of positive and death cases all over the world could generate erroneous predictions. So, it is required for the studies to comprehend the outbreak at regional, local, and international levels to create accurate predictions. Analysts would also require information from health specialists that
Fig. 4 Nature, advantage as well as disadvantage of clinical data

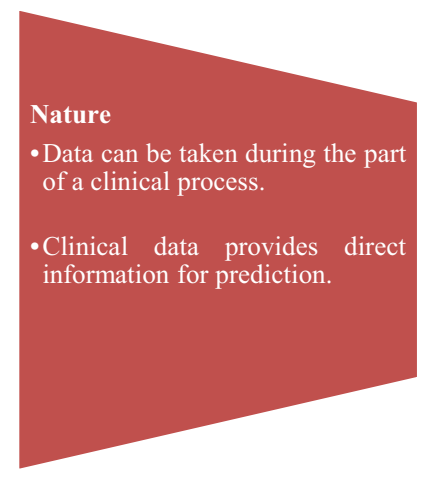

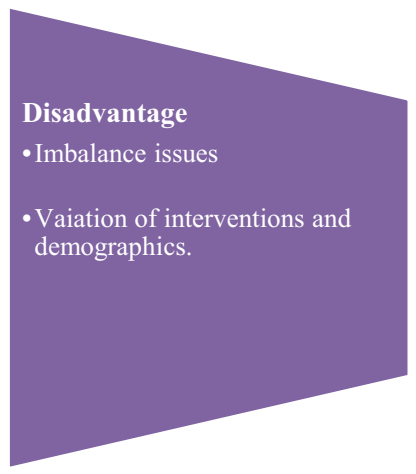


Fig. 5 Nature, advantage and disadvantage of online data


differentiate among deaths that happened due to COVID-19 and deaths might have caused due to some other unknown disease. This particular information may not be available in the majority of the study. So, it is complex to verify the accurate level to which citizens follow the government measures. The presence of huge missing values that may affect the analysis is one more challenge of online data. These issues need to be measured to make a correct prediction of COVID-19 using online data. Figure 5 briefly depicted about the advantages as well as disadvantages of online data respectively.

\subsubsection{Biomedical data}

As mentioned in the literature, the majority of the research for the detection, classification, and prediction of COVID-19 has been brought on the medical image data set. Selection of medical images like X-ray or CT images is taking as another solution to defeat the deficiency of RT-PCR supply. This is due to the proper analysis of the medical image provides an exact prediction of COVID-19. Still, there are a few limitations that need to be considering in the utilization of the medical image dataset. Though the COVID-19 related data has been considering from biomedical data, the categorization of medical images became the major challenge in health diagnosis because of partial medial images. Due to the frequent imagery distinctiveness amid COVID-19 as well as other kinds of pneumonia, the procedure of automatic division became complex in
CT images. The complexity of early identification has highlighted the presence of no or mild $\mathrm{CT}$ results in many early cases of infection. Also, the CT scan equipment is exclusive and patients are depicted to high radiation. Though chest X-ray images are inexpensive and depicted to low radiation, these images have a superior false analysis rate. Its advantages and disadvantages along with the nature are depicted in the following Fig. 6 respectively.

\subsubsection{Impact of data in COVID research}

Progress of exact prediction models for the epidemic is crucial to offer insights into the spread and results of this contagious COVID disease. For COVID related cases, we require natural clinical data rather than online data. One can analyze that, usual epidemiological techniques may be efficient and consistent only if, the social connections are inactive through time. Due to the large scale as well as difficulty nature of the problem in enhancing epidemiological techniques, ML and DL have attained an attraction for constructing epidemic prediction models. The ML approach intends to improve techniques with advanced generalization capability and better prediction consistency for longer lead-instances. The usage of ML in processed information (data) predicts the explicit information. Time-series data can be considered as input to ML algorithms as it reflects the modifications that an incident has undergone over time [28]. In the same way, AI involved health imaging
Fig. 6 Nature, advantage and disadvantage of biomedical data

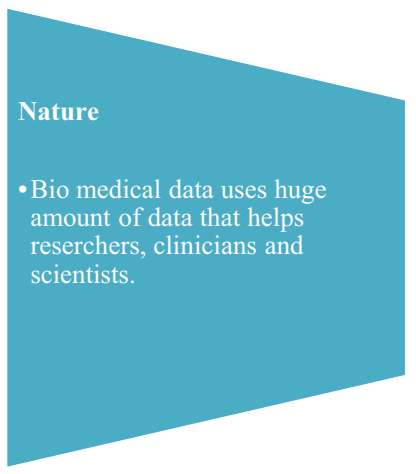

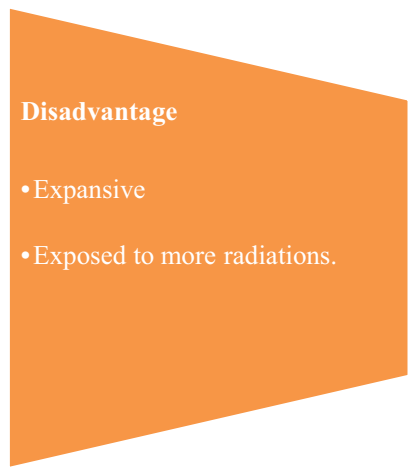


DL models has been enhanced in image attribute extraction and spatial relation attributes. So, Ni et al. [83] have assumed that DL models might be capable in identify quantitative lessons as well as feature extractions by using chest CT images and presented a comparison of its quantitative resolving performance using radiological residents. Tuli et al. [110] has highlighted an efficient cloud framework to predict the disease. They showed that the usage of ML-based enhanced technique has predicted the threat of pandemic in various countries globally. They proved the enhanced accuracy contrasted to baseline techniques and also emphasized the emerging trends. As it is an aware situation that the pandemic has presented a huge amount of challenges to clinical trials and medical research. Zame et al. [111] has identified few challenges such as current clinical trials for treatments of Covid-19, non COVID-19 drugs, as well as new medicines to treat pandemic and advised methods in which ML can assist in response to them. As mentioned previously, CXR and CT scan are the standard image analysis for pneumonia, due to the nature of CXR relays cheaper, widespread, and faster when compared to CT images. Pereira et al. [88] has noticed pneumonia originated by COVID-19 from other kind's as well as healthy lungs with only CXR images. So to attain this intention, the authors have introduced a categorization schema with the hierarchical and multi-class perceptions, because pneumonia can be controlled as a hierarchy. In this way, several types of inputs such as lung image dataset, detection related dataset, information from the publicly available datasets etc. are used to the ML and DL models for the prediction of COVID-19.

\subsubsection{Challenges in Processing COVID-19 data using intelligent computing approaches}

The outbreak of COVID-19 pandemic has impacted the lives of billions of people all over the world economically and socially. The pandemic has instigated the scientific community to intelligent computing approaches in the identification, prevention and assessment of COVID-19, as these techniques have been prominently used in various applications varying from bio-informatics to image processing. For the effective implementation of scientific methods, data plays a main role, which is available either in the form of closed-source or opensource. The possession of the scientific contribution has been protected using closed data while the open-source data provides high quality, transparency and versatility $[112,113]$. In the current COVID-19 pandemic, the usage of open-source data is considered to be more adequate in the diagnosis of COVID-19. Therefore, the combination of intelligent computing approaches and open-source data contributes effective solutions for the diagnosis and prediction of COVID-19 from the textual data such as COVID-19 case reports and social medial data and from medical images such as chest X-ray and computer topography scans. The usage of intelligent computing approaches in the diagnosis of COVID-19 from medical images can overcome the limitations of reverse transcriptase polymerase chain reaction (RT-PCR) test kits, while the predictions of virus transmissions can be assessed using epidemiological analysis of COVID-19 case reports. Furthermore, the mining of social data provides the policy makers with socio-economic analysis.

Though the usage of intelligent computing approaches and open source data provides effective solutions to the diagnosis of COVID-19 pandemic, there exist many challenges in the usage of COVID-19 open source data. One of the key challenges is to improve the accuracy of medical images for its application in clinical practices as most of the researchers suggested further improvement of accuracy of COVID-19 diagnosis from the medical images. The patients tested positive with RT-PCR can have a normal chest scan at the time of admission. Due to this, researchers have indicated that RTPCR only remains as the primary source of identification, while the medical imaging serves as the secondary diagnosis method [114]. Therefore, another challenge exist in establishing the correlation between radiographic and RT-PCR tests $[115,116]$. Other challenge exists in the development of contact-less work-flows to protect the medical practitioner from COVID-19 infected patients [117]. Most of the datasets used in the diagnosis are smaller in size. Therefore, to provide better observations and accuracy using deep learning approaches, larger datasets are required. The effective epidemiological analysis of COVID-19 can be performed by considering the textual data from online sources. The analysis of these epidemiological results is not accurate as most of the studies considered only a few determinants. Therefore, to make accurate predictions it is fundamental for the research analyst to find out the dissemination of virus at regional, national and international level. Moreover, to make accurate prediction of COVID-19 using textual data, factors such as implementation of quarantine measures, distinction of deaths caused by coronavirus and deaths caused by anonymous disease must be considered.

\section{Critical analysis}

It is known that DL is a subset of ML. In fact, DL is exactly like ML as it functions in the same way. Still, it has some different abilities like necessary ML models do become gradually superior at whatever their function is, and they still require some assistance whereas a DL model is capable to learn through itself without any guidance. Like other real world applications, ML and DL have become a rapidly growing trend in the field of healthcare. Sensors in wearable components present actual patient data such as health condition, blood pressure (BP), heart rate, and other major parameters. Health specialists can utilize this data to identify the health of 
each person and can easily predict the incidence of any diseases in the future. ML and DL techniques are distributing novel affectivities and chances, allowing researchers, physicians, and clinicians to easily predict and understand the diseases and progress the lives of others. The usage of ML, DL, and other mathematical models in predicting harmful diseases along with their percentages, graphs, best outcomes, publication rates, statistics, and country-wise research data and many more are explained briefly in the following subsections respectively.

\subsection{Most popularly used intelligent methods for COVID-19}

This subsection describes the mostly used methodologies of ML and DL Models for COVID-19 related issues. An indepth analysis has been conducted and the reasons behind the usage of these particular methods as well as their statistics have been mentioned in Figs. (7, 8, 9, and 10).

\subsubsection{Popular ML methods}

Out of all the considered articles used in this research work, we found many ML articles have been used for solving COVID-19 conflicts. Based on these ML articles, we made an analysis on the usage levels of ML methods among them. Various methods such as SVM, RF, LR, LIR, ANFIS, MLP, KNN, NB etc. were found and their statistics have been mentioned in Fig. 7.

From the analysis made for Fig. 7, we have observed the ML techniques such as RF, SVM, LR, MLP, KNN as well as NB have been used mostly for COVID-19 which is the top six

\section{$\%$ of types of various ML techniques for Covid-19}

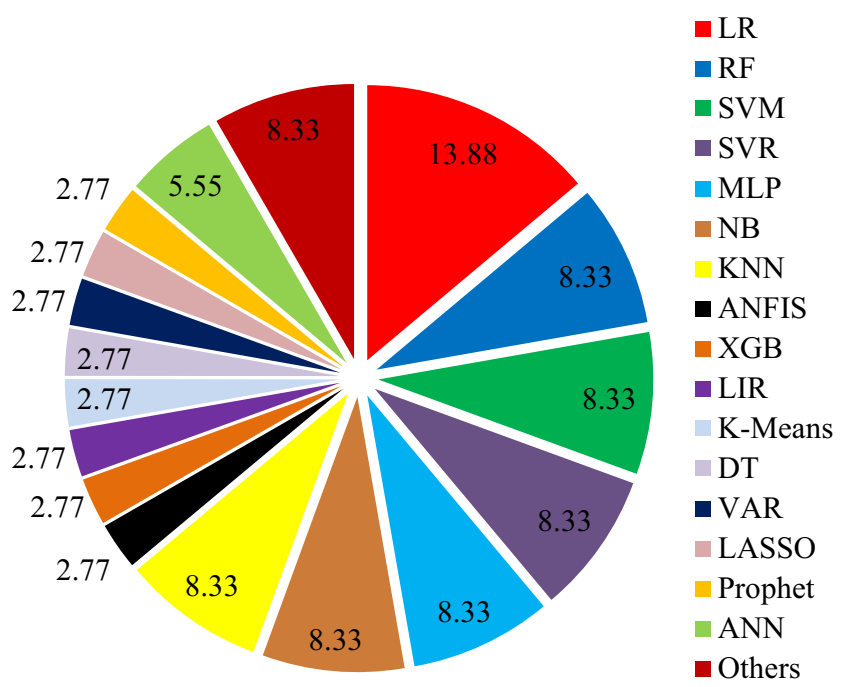

Fig. 7 Usage levels of several ML methods for COVID-19

\section{$\%$ of types of DL techniques for Covid-19}

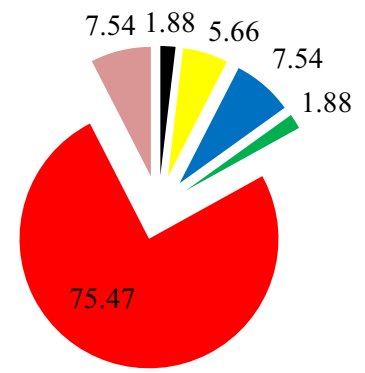

- Encoder-Decoder
LSTM
- GAN
RNN
- CNN
Others

Fig. 8 Usage levels of several DL techniques for COVID-19

among other ML methods. The main intentions behind the utilization of ML techniques mentioned in Fig. 7 have been observed and described in our point of view. SVM's have been used for classification of features mostly. It has also produced an effective accuracy rate in many applications, so that SVM has been used for COVID-19 related issues. Mainly, it is also noticed that SVM is a strong binary classifier. Another ML technique named ANFIS has also been used. The key motto to use this method has been observed such as; it is an effective method that combines NN with Fuzzy Logic (FL). Therefore, ANFIS has been used to cope with fuzzy data of the COVID-19 outbreak. Moreover, it is also noticeable that algorithms such as AdaBoost, XGB, NB, etc. are used for novel COVID-19 data fitting and thereafter for classifying the same data. Also, methods such as LASSO (least absolute shrinkage and selection operator), RF have been used for fitness of COVID-19 related data as they are proved to be efficient in dealing with fitness function problems. One of the known methods named decision tree has been used mostly for the COVID-19 genomes classification as it has proved its effectiveness in classifying modules. It has also been used for the minimization of several calls as well as for maintaining the number of clusters of COVID-19 data. K-Means algorithm has proved its efficacy for using in data-driven analysis, so that research has been performed for grouping of heterogeneous elements into clusters of COVID-19 data. Moreover,

\section{Top 3 variants of CNN used for Covid- 19}
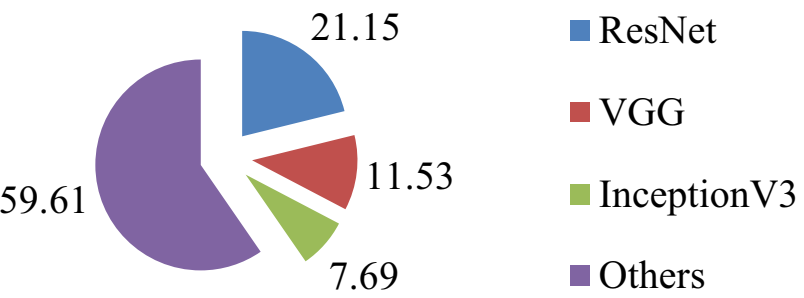

Fig. 9 Usage levels of top3 variants of $\mathrm{CNN}$ among others 
$\%$ of rate of publication of ML \& DL for Covid-19

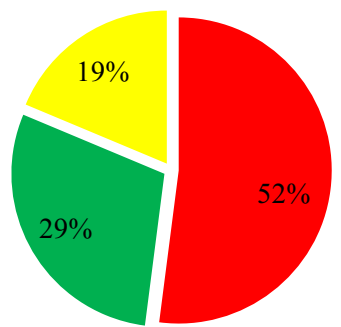

- Deep Learning

- Machine Learning

Others

Fig. 10 Publication rates of ML, DL with others necessary articles used for study

some other ML techniques such as LR, VAR have been used frequently in many applications, so that those have been utilized for regression analysis of COVID-19 data and predicting as well as forecasting of COVID-19 time series data respectively. The type of neural network named MLP has proved its effectiveness in information processing in many types of research. Therefore, this method has been applied to predicting the outbreak of COVID-19. In this way, as per our perception, we have mentioned key reasons behind the usage of every technique found in this research work.

\subsubsection{Popular DL methods}

Similarly, for DL techniques, we made an analysis on the usage levels on the mostly used ones among them. It is observed that various methods such as CNN, RNN, and GAN, LSTM, Auto Encoder Decoder etc. have been mostly used and their usage levels statistics have been mentioned in Fig. 8.

We have observed that CNNs are the mostly used DL techniques for resolving COVID-19 related problems. The main architecture of CNN is the popular DL technique with higher attainment in the medical imaging field. The capability to learning characteristics from exact domain images is the main achievement of CNN. This technique is simple and faster to use without the requirement of a vast annotated dataset for training. Due to this reason, many researchers have shown their interest in using this method, particularly with medical imaging. CNN's are reciprocal to image data and is the reason for being used in COVID-19 related issues. As the control of COVID-19 lies on fast detection, CNN proved as having a higher capability to diagnose medical images with high accurateness. Also, we found that many variants of $\mathrm{CNN}$ have been used and analysis of its usage levels has been performed. From the analysis on the usage levels of $\mathrm{CNN}$, we found that variants such as ResNet-16, ResNet-18, U-Net, VGG, DenseNet, GoogleNet, AlexNet, InceptionV3, MobileNet, VBNet, Squeeze Net, etc. have been used. The top three CNN variants mostly used for COVID-19 related conflicts are ResNet, VGG, Inception variants. The reason behind the usage of these three variants mostly is due to its effectiveness in identifying the COVID-19 patients with chest X-ray radiographs. The technique of Residual neural network (ResNet) is an enhanced version of CNN that includes shortcuts among layers to resolve a problem. ResNet which is known to be an improved DL architecture is moderately simple to optimize and can get advanced accuracy. Visual Geometry Group Network (VGG) is another improved version of CNN that executes well on the ImageNet. VGGNet uses smaller filters to have enhanced image extraction functionality. InceptionV3 is a type of $\mathrm{CNN}$ technique. It comprises of several difficulties and utmost pooling steps. This network has various symmetrical as well as asymmetrical structure blocks, where every block includes average and max pooling, convolution branches, dropouts, concatenated as well as fully combined layers. This analysis of the usage levels of the top three variants of CNN is mentioned in Fig. 9.

Moreover, it is also observed that RNN, as well as GAN, are mostly used methods after CNN. To evaluate the transmission development at the initial and predict the upcoming possibilities of the spread, RNN can be adapted with long shortterm memory (LSTM) cells. The advantage of this model is that, it permits the technique to remember earlier data better to make more exact predictions. Prediction of COVID-19's positive, negative, released, and deceased cases are attained with RNN. Generative adversarial networks (GANs) are a type of $\mathrm{NN}$ where two networks learn at a time, one concentrates on discrimination and other on image generation. GANs have produced huge development and incredible performance in several applications like image synthesis, classification and pattern transfer etc. With these advanced methods, the identification of this kind of pandemic in the initial stage will assist in quick recovery and releasing the stress of healthcare systems. It is known that LSTM is an efficient DL approach, which is suitable for the prediction of time series sequential data [63]. LSTM covers the limitation of context based prediction of traditional RNN and it can standardize ascent-flow of data. It also has the ability to store past data in hidden states, so that the prediction rate of LSTM is more accurate. Due to this nature of LSTM, it has been used for prediction as well as forecasting of COVID-19 data. GRU is similar to LSTM. But, in comparison with LSTM, GRU has greater convergence rate and due to this nature GRU has also been used for COVID-19 verification with the help of original COVID-19 data.

\subsection{Publication analysis of $\mathrm{DL}, \mathrm{ML}$ and similar methods}

In this research work, in order to extract related research articles, different keyword ("COVID 19", "Machine Learning for COVID-19"; "Deep Learning for COVID-19") searches have been performed in the standard online databases named "Google Scholar, IEEE Xplore, Elsevier, Springer". Based on the keyword searches, we found that 795 papers were 
related to COVID-19. Among those 893 papers, we have excluded those articles not related to our review work and found them to be 672 papers. After exclusion, 123 articles are selected as related ones for the study. Apart from these 123 papers, we have also cited another 23 resources related to COVID-19 such as datasets and nature of data used for COVID-19 etc. The total number of articles used in this research is 123. Purely on the basis of these articles found, percentage analysis has been made on the usage levels of ML, DL among other COVID-19 related papers used for this study and the analysis has been depicted in Fig. 10.

The individual articles related to ML, DL as well as other applications have been depicted in Fig. 11. All the selected articles are extracted from the Google Scholar and other standard databases and articles published from December 2019 to September 2020.

\subsection{Analysis on publications of only ML, DL \& mathematical model}

In the previous section, we mentioned a number of research articles from Dec 2019 to September 2020 based on the keyword search as well as filtering. In this section, we calculated the rate of published articles only in the area of ML, DL, and Mathematical Models. The papers considered for this study are 123 . Among those 123 papers, we found that 36 papers are related to ML, 64 are for DL and 30 other related papers respectively. Therefore, based on these 123 papers, the rate of publication of articles related to only ML, DL \& others are taken and depicted in Fig. 12. In Fig. 12, major percentage (89\%) of COVID-19 publications is clinical as well as disease behavioral based and non-intelligent based methods, whereas $7 \%$ articles of the total database is concentrated on DL and $4 \%$ of articles are based on ML models. It shows the relevance of COVID-19 publications are mainly based on the study of various symptoms related to COVID-19, financial issues,

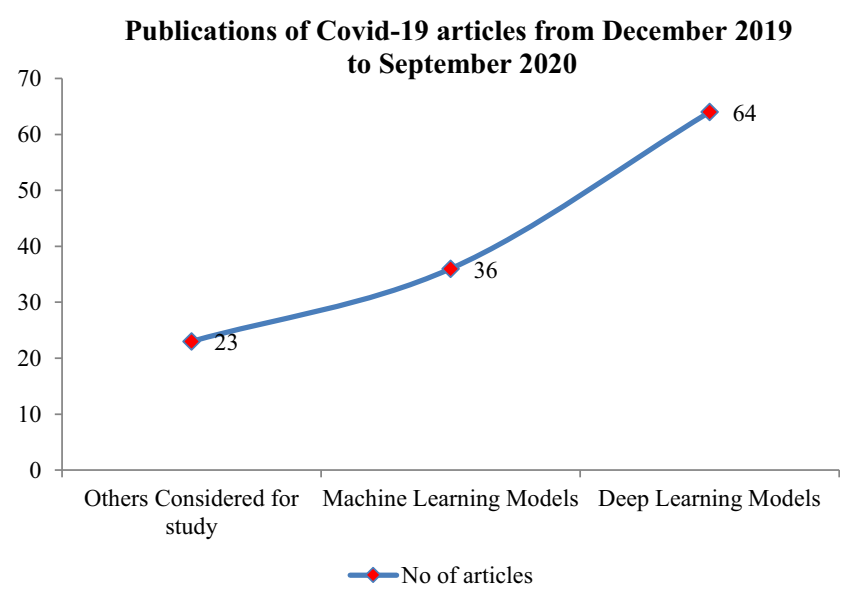

Fig. 11 No of publications of COVID articles from Dec 2019 to Sep 2020

\section{$\%$ of rate of publication among ML, DL \& other} articles

- Others Deep Learning Models $\square$ Machine Learning Models

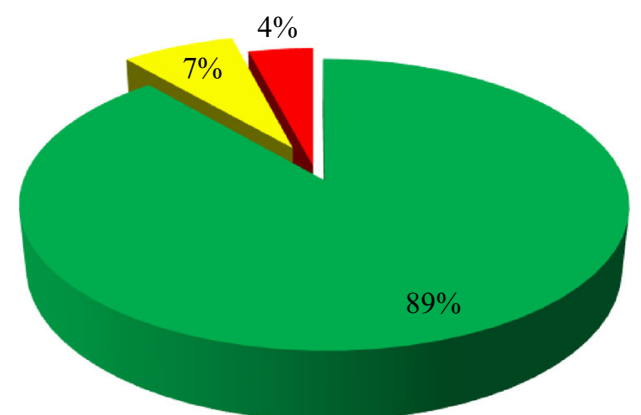

Fig. 12 Rate of publication of only ML, DL \& other COVID-19 related articles

hospital management and patient related research, drug discovery, clinical diagnosis etc. other than intelligent based methods. However, it is worthy to note that sooner the disease will be in its old state, more intelligent computing based research will be carried out for fruitful intelligent solutions.

\subsection{Analysis on publications of articles related to COVID-19 prediction and detection}

Due to COVID-19 out broke in a short gap, most of the researchers have made their research in detection, identification as well as prediction of COVID-19 by using advanced ML and DL techniques. Out of our all gathered articles, we found that major researchers have published detection based articles followed by prediction based research. In this section, we divided prediction based; detection based as well as identification based articles and calculated their publication frequency accordingly. From our analysis, we got $31 \%$ of articles belong to the detection of COVID-19, 27\% of articles are for the prediction of COVID-19 and 17\% of articles are for the screening \& assessment of COVID-19 using intelligent approaches as top three article types respectively. This analysis has been depicted in the Fig. 13 .

Moreover, as this subsection mainly focuses on prediction and detection based articles, analysis on the number of papers used in this study has been conducted and the results have been obtained and depicted in Fig. 14.

\subsection{Impact of ML/DL in COVID-19 predictions}

Since the outbreak of COVID in January 2020, many research works are conducted and are being performed by several researchers for detecting, forecasting, predicting, the data of active, death, and recovered cases of COVID-19 throughout the world. According to our study in this paper, we found that most of those research works are mainly focused on utilizing 


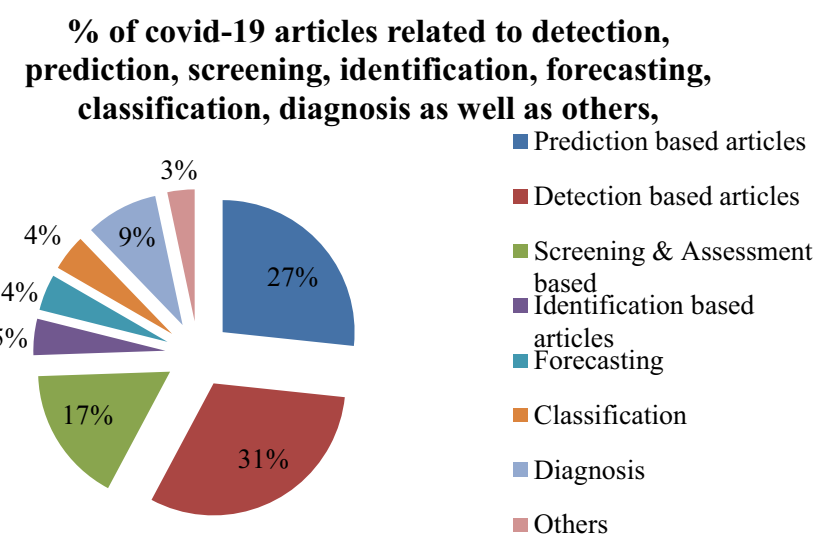

Fig. $13 \%$ of rate of publications of Prediction, detection \& identification, screening, forecasting, classification, diagnosis based articles used in this research

machine learning, deep learning, as well as mathematical models. Several models have proved their effectiveness in forecasting and predicting the COVID-19 by applying them on available COVID-19 related information. Still, if the basic nature of the COVID-19 pandemic is seen as a frightful problem, the predictive nature of the future is irregular. Indecision is deep-seated in many people who are unfamiliar with the infectious virus and the multifarious, dissimilar and vibrant human deeds, government involvement as well as testing circumstances. The utmost ambiguity of the situation can mislead the objective of prediction accuracy. In this subsection, we have deeply focused on exploring the monitoring capability of predictions and some of the observations on the forecasting of COVID-19 data, mentioned in Table 3.

Many advanced research works have been observed, however, no research work will help to predict the exact scenario of the condition of pandemic COVID-19 under these following criteria. Many researchers have mentioned in their research works that, the cases of corona will not stop up to the end month of August, 2020 and the impact rate will be high. Later, after the ending of the August month, the impact rate will drastically decrease and nothing will happen. In the same

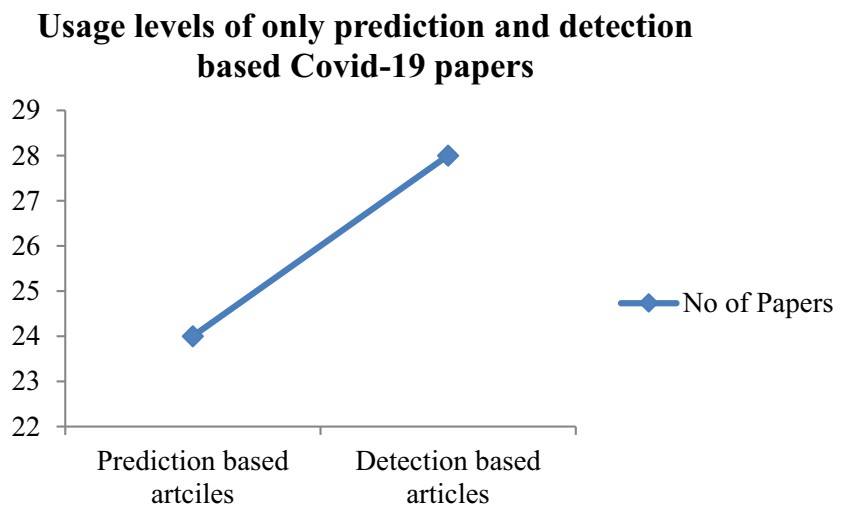

Fig. 14 No of prediction \& detection based papers obtained
Table 3 List of problems focused by various Forecast models for COVID-19

\begin{tabular}{llll}
\hline S.No & Focused Problem & Model used & Reference \\
\hline 1 & COVID-19 Future Forecasting & ML & {$[25]$} \\
2 & COVID-19 Forecasting & LSTM & {$[63]$} \\
3 & Forecasting of COVID-19 cases & DL & {$[66]$} \\
\hline
\end{tabular}

way, in a country like Singapore, Prime Minister Hsien-Loong Lee has declared the extension of the circuit breaker to June 1 in reaction to the point of COVID-19 cases on April 21. But, such planning and decisions can be efficient by well significant where country like India, is in its epidemic life cycle, when the rotating point is imminent if it has yet come, and when the epidemic will stop. Modifications may be created according to the changes in the opinions and predictions on these facades. But we can't predict the exact scenario of what will happen in upcoming days. Nobody in this world is sure whether the exact positive cases will increase or decrease! The exact prediction rate will be found based on the number of tests done. No country is aware of the overall count of people affected by COVID-19. All we know is the status of infectious people who have been officially tested. All those patients who have a lab-authenticated infection are considered as confirmed cases. This means that the counts of validated cases relay on actual tests of a country. There won't be any data without testing. With no information on who is affected by the virus, we have no state of understanding the epidemic. This leads to the failure of intelligent prediction models. The training data to any intelligent based models should be accurate, so that the test results can be efficiently validated. We should collect and maintain the data in a continuous as well as appropriate manner. But, this is not happening correctly in many countries due to their policies.

Another reason for inaccurate predictions is the immigration problem. For an instance, it is known that many citizens have migrated from India to other states and countries for jobs and study purposes. Due to these epidemic situations, all the citizens of India who are staying outside are moving to their own country. Even though India is migrating its citizens to their own country from other countries. But, they are not maintaining the number of tests with the newly migrating people. This is the same situation facing all the countries. Out of several tests done for people, they are not considering such type of migrating people, and hence data is not natural, which is a basic problem. In this type of situation, no prediction model will function accurately due to unnatural data. Generally, our default anticipation in predictive scrutinizing is that predictions will vary, particularly when the actual world situations like human behaviors, government policies, testing protocols, etc. are also quickly changing. In such cases, we 
must not suppose the prediction model trained with the information as of at the moment to be accurate for a diverse scenario later. When allowing for the dynamics of individual behaviors and government strategies and other existing world scenarios that the training data, as well as the mathematical model, cannot represent accurately, forecasting supervising would be an extra expensive exercise. The data widely accessible today is based on tests, which are taken differently in several nations. They need not essentially signify the entire infection description which is the hypothetical input of the model. The future is always unsure. We should make it note while predicting. No country and no researcher had predicted the COVID-19 spread earlier. With accepting the indecisive nature of the continuing COVID-19 epidemic and our rising difficult as well as the consistent world, what is ultimately and essentially required are the elasticity, robustness, and flexibility of citizens, associations and governments, as well as distribution and management, to deal with erratic and unnecessary future events.

\subsection{Analysis of ML \& DL with image data}

Almost every year since 2012 has given us big breakthroughs in increasing ML and DL models for the task of image classification. The easiest way to get image data is to get them from pre-existing sources. In this research work, we thoroughly checked whether these all papers belong to ML as well as DL or not and we have divided these papers separately based on the type of inputs such as image data, text data as well as others. Based on this, we calculated the percentages of 89 articles as a total no. of papers out of a hundred percentages. After calculating the percentage, it is observed that $60 \%$ of articles having image data as input, $31 \%$ of articles have text data and the rest of the $9 \%$ of articles have other data types. This overall analysis has been depicted below in Fig. 15. It is also observed that X-ray, as well as $\mathrm{CT}$ images, are mostly

\section{$\%$ of rate of publication of Covid-19 articles with several types of Inputs}
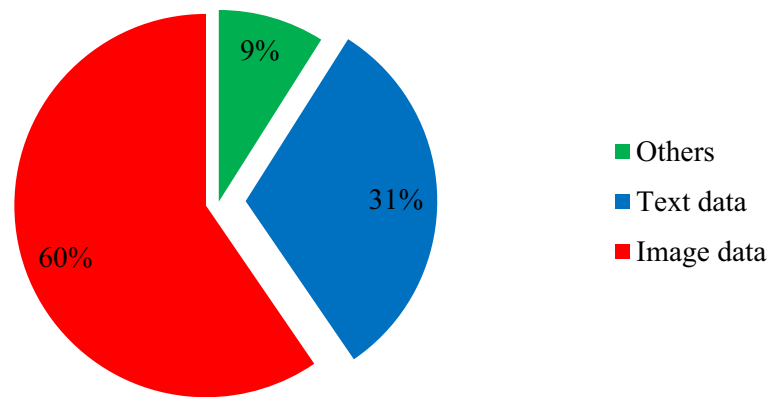

Fig. 15 COVID-19 articles publication rate having various types of inputs used as input data. The analysis of articles having different input image data along with others has been shown in Fig. 16.

Moreover, it is obtained from the analysis that 67 papers are related to COVID-19 with images as input. Among those 67 papers, 19 papers are related to CTR images, and the rest of 34 papers are related to $\mathrm{X}$-ray image data as inputs. This analysis has been depicted in Fig. 17.

\subsection{Some efficient ML and DL based Approaches for COVID-19}

In this research work, we found many articles that have the best accuracy related to ML and DL based methods. In this subsection, papers with 5 best outcomes (real impactful research) have considered and been explained in a brief manner. These papers have been selected based on a strategy and we named that strategy as "strategy 1". This strategy 1 includes the impact of accuracy rate, strength of the type of methods used, etc. Our strategy 1 has been created to examine why the considered five papers taken as top five among others. Also, we gave higher priority to the papers having a high accuracy rate.

We selected the paper contributed by Das et al. [81] as the top one best paper on the basis of our strategy 1 . The highlights of the paper which satisfies our strategy 1 are as follows: In general,

- An advanced deep learning methodology named Inception net has been developed.

- Six unique image datasets of COVID-19 patients having positive cases, tuberculosis health issues etc. are considered.

- Classification metrics such as accuracy, sensitivity, precision, etc. are considered.

Satisfied requirements of our strategy 1.

- Classification performance has been improved by incorporation of Inception Net.

- Inception Net has successfully classified healthy as well as non healthy cases which proved it as powerful classifier.

\% of Types of Images used as input along with others
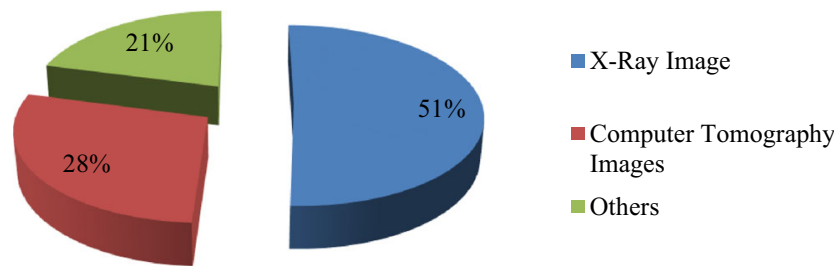

Fig. 16 Usage levels of types of input image data considered for this study 
Usage levels of papers with image data

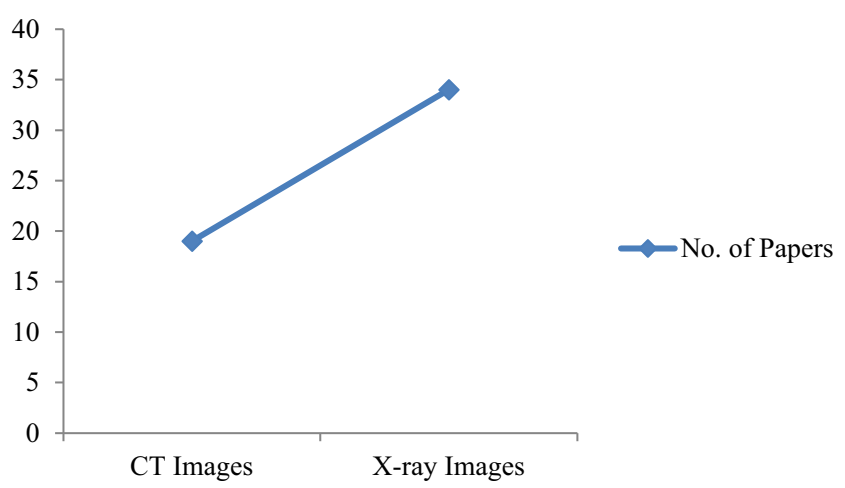

Fig. 17 No. of papers with image data used as input considered in this study

- Although the considered data is heavy, Inception net achieved $99.96 \%$ of classification accuracy.

- The method is helpful in classifying COVID-19 CT images.

Similarly, the second-best paper selected is contributed by Rahimzadeh and Attar [98] based on our strategy 1 . The highlights of the paper which satisfies our strategy 1 are as follows: In general,

- Advanced deep learning methodologies which are variants of CNN named Xception, ResNet50V2, and Concatenated Network have been used.

- As CNN's are reciprocal to image data, the developed CNN architectures have been applied on Chest X-ray dataset of patients having COVID-19 of $180 \mathrm{X}$-ray images and 42 images with pneumonia.

- Xception and ResNet50V2 deep learning models have been adopted and better accuracy rate has been obtained by concatenating the features of outcomes of both the models.

- The proposed methods helped in detecting the COVID-19 among the three types of image samples such as normal persons, patients with pneumonia as well as patients with COVID-19.

- This concatenated architecture may be helpful for accurate medical diagnosis with the help of complex data.

Satisfied requirements of our strategy 1 .

- To check the effectiveness of proposed NN architectures, they have been applied to 11,302 images further.

- An accuracy of $99.50 \%$ has been obtained for detecting COVID-19 from the X-ray images.

The third-best selected paper is contributed by Toğaçar et al. [97] based on strategy $\mathbf{1}$. The highlights of the paper which satisfies our strategy 1 are as follows:
In general,

- Deep learning models such as mobile Net V2, squeeze Net are has been considered.

- Dataset contain 3 image classes such as pneumonia, normal as well as corona virus X-rays.

- Fuzzy coloring techniques has employed for preprocessing.

Satisfied requirements of our strategy 1.

- To process feature set, a powerful optimization algorithms named SMO (Social Mimic Optimization) has employed and better results are observed.

- Standard ML classifiers named SVM has employed to combine and classify the features.

- By utilizing above methods, an accuracy of $99.27 \%$ has been achieved for diagnosing COVID-19.

Based on strategy 1, the fourth-best paper selected is contributed by Apostolopoulos et al. [85] based on strategy 1 . The highlights of the paper which satisfies our strategy 1 are as follows:

In general,

- An effective variant of CNN named Mobile Net V2 has been considered.

- Dataset having 3905 X-ray images has been considered.

- For detecting CT images variants of CNN such as AlexNet, VGGNet16, VGGNet19, GoogleNet, as well as ResNet50 have been selected.

- The method is helpful for representing the COVID-19 pneumonia-related opacities among various types of samples such as Fibrosis, Effusion, Pneumonia, Pleural thickening, Cardiomegaly, Nodule, Mass and Hernia using CXR.

- The prediction rate has been improved with the incorporation of combination of transfer learning with self features, fine-tuning and from scratch respectively.

- The model explored higher accuracy which is inexpensive and rapid for detecting COVID-19 using CXR.

Satisfied requirements of our strategy 1.

- $99.18 \%$ of classification accuracy has been obtained for diagnosing COVID-19.

The final best paper selected is contributed by Apostolopoulos and Mpesiana [17] based on our strategy 1. The highlights of the paper which satisfies our strategy 1 are as follows:

In general,

- Intelligence transfer learning procedure is adopted. 
- As CNN's are reciprocal to image data, the developed CNN architectures have been applied on Chest X-ray dataset of patients having COVID-19 of 1427 X-ray images and 700 images with pneumonia.

- $\quad$ The proposed method helped in detecting the COVID-19 among the three types of samples such as normal persons, patients with pneumonia as well as patients with COVID19.

- This transfer learning may be helpful for accurate medical diagnosis with the help of complex data.

Satisfied requirements of our strategy 1.

- Detection accuracy for COVID-19 has enhanced by incorporating the standard CNN's like MobileNet for transfer learning.

- An accuracy of $96.78 \%$ has been obtained for detecting COVID-19 from the X-ray images.

\subsection{Country wise research contribution}

As we know that ML, DL, and other mathematical models have proved their efficiency in predicting COVID-19 in tremendous way, so to make it more successful, different authors from different countries have contributed their research regarding COVID-19. As per our analysis, the spread of COVID has affected many countries where all these countries are located in various continents like 54 countries are situated in Africa, 48 countries are in Asia, 44 countries are in Europe, 33 countries in Latin America and the Caribbean, 14 in Australia and 2 in Northern America respectively. Among these countries, 656 authors from 6 continents have contributed their research on COVID-19 by using ML and DL Models. 25, 345, 129, 17, 12 and 128 authors from Africa, Asia, Europe, Latin America, Australia, and North America have made their research with these techniques and also got better results. Out of all these continents, Asia has got the highest number of authors who have published their research papers regarding COVID-19 by using ML and DL. Out of all these countries, China has placed on a first position by having a contribution of 163 author's research papers followed by USA and India with the contribution of 113 as well as 63 authors' research articles respectively. This huge continent wise analysis has been shown in Fig. 18. Kiribati, Marshall Islands, Micronesia, Nauru, Palau, Samoa, Solomon Islands, Tonga, Tuvalu, Vanuatu as well as some other countries such as Melanesia which is a sub-region of Vanuatu and Polynesia which is sub-region of Samoa are the countries that are not affected by COVID-19 till now ${ }^{1}$.

Similarly, continent wise data has been collected and analyses separately and depicted in the Figs. (19, 20, 21, 22, 23, and 24) respectively.
$\%$ of rate of no of authors invovled in Covid-19 related research in Continent Wise

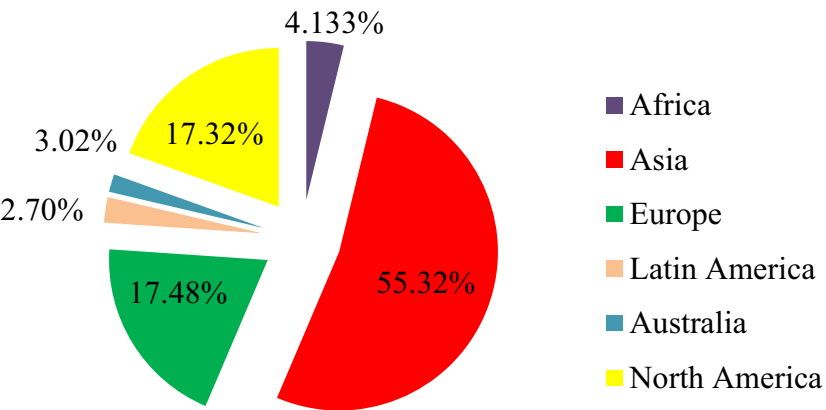

Fig. $18 \%$ of ratio of no. author's contribution worldwide

From the Fig. 19 and 345 authors with the highest number from 16 countries in the Asian continent have contributed their research articles regarding COVID-19 by using various intelligent models.

Similarly, 129 authors from 16 countries in the European continent have contributed their research articles on COVID19 with ML, DL, and Others. We depicted this scenario in Fig. 20

Here, in Fig. 21, a total of 17 authors from 3 different countries in Latin America have contributed their research on COVID-19.

12 researchers from the Australia continent have contributed their research on COVID-19 with ML, DL, and other Others respectively and this scenario is depicted in the above Fig. 22 respectively.

Here, 128 researchers from two countries like the USA and Canada have contributed their research on COVID-19 with ML, DL, and others which are depicted in above Fig. 23. Similarly, 25 researchers from Egypt as well as Algeria from African continent has contributed their work on COVID-19 with ML, DL as well as others and is depicted in the following Fig. 24 respectively.

\subsection{Existing survey comparisons}

In this section, an analysis between the existing survey and this present research is made on the basis of some criterion. The comparison has been made based on the following criterions:

- Is critically reviewed or not reviewed?

- What type of Intelligent method focused?

- What type of data used for covid-19 is observed in survey?

- Whether followed systematic literature process or not followed?

On the basis of considered criterions, a comparison has been made with existing surveys and it is worthy to note that, only intelligent machine learning techniques are focused and 
Fig. 19 Usage levels of no of author's contribution for COVIDrelated articles using ML, DL \& Others in Asia continent
No of authors Contributed in Countrywise in Asia

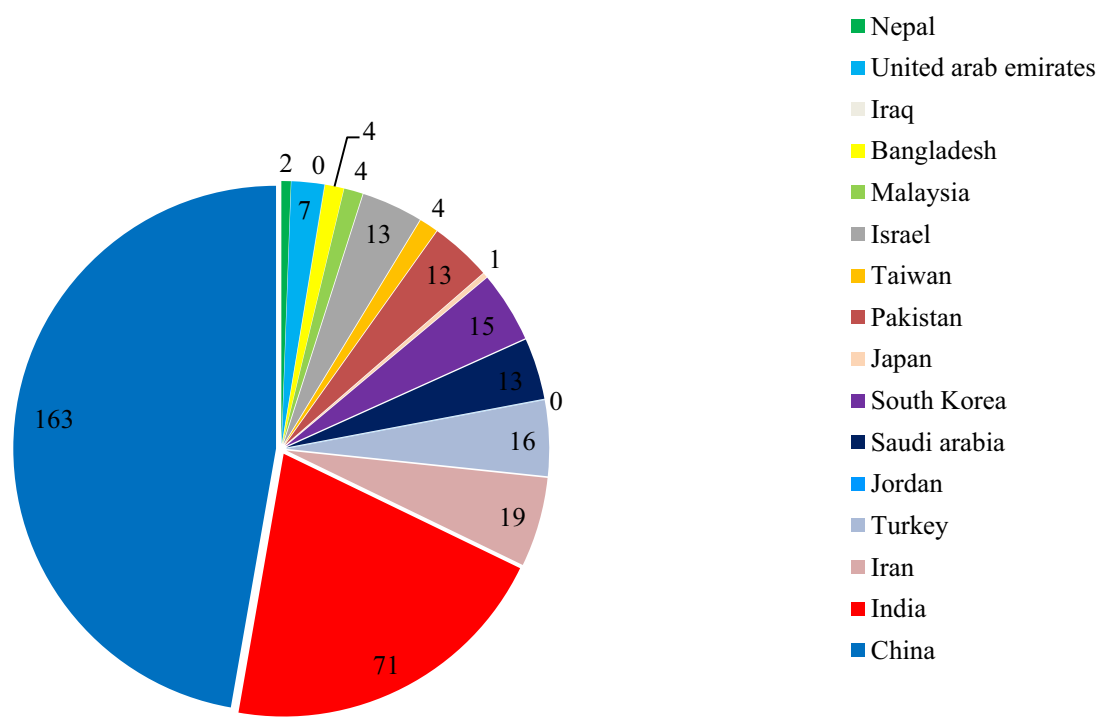

mostly text data (in the form of datasets) has been utilized. Therefore, these became limitations for existing survey and almost all the limitations are addressed and covered in this present study. The existing surveys comparison with this present study is depicted in Table 4.

\section{Sectors effected due to pandemic COVID-19}

In this subsection, the sectors that are already affected due to COVID-19 throughout the world have been mentioned. The main intention of this subsection is to give an overview on several affected sectors to the researchers as well as

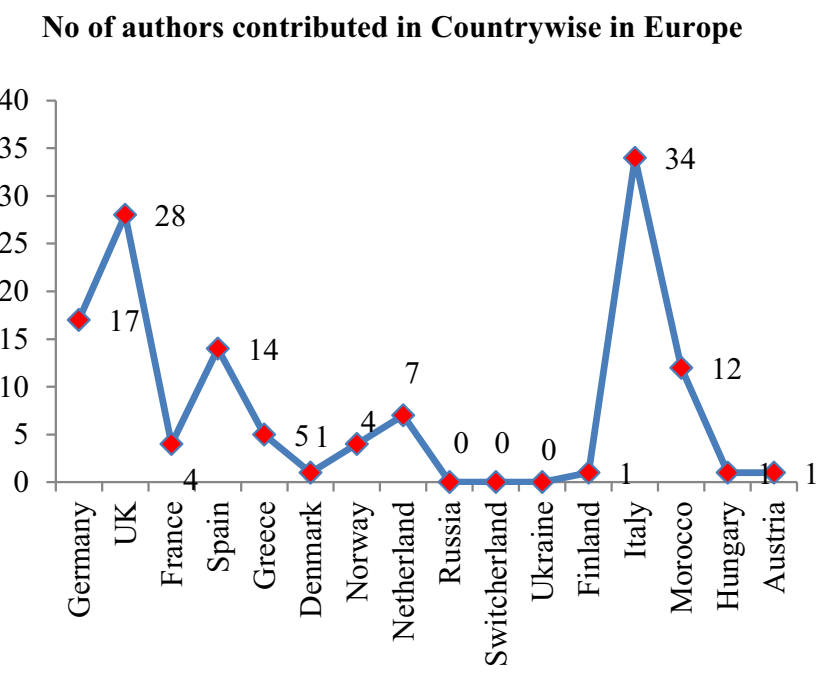

$$
\leadsto \text { No of Authors }
$$

Fig. 20 Usage levels of no. of author's contribution for COVID-related articles using ML, DL \& Others in Europe practitioners and others. It will be helpful to analyze these areas using intelligent methods to build effective solutions. A brief analysis has been conducted from multiple online resources especially the website statistica.com. It is observed that throughout the world, many sectors such as agriculture sector, chemicals \& resources sector, construction, consumer goods \& FMCG, E-Commerce, energy \& environmental services, finance sector, insurance sector, real estate sector, health \& pharmaceuticals, internet, media \& advertising, metals \& electronics, retail\& trade, sports sector, transportation \& logistics, travel, tourism, hospitality, etc. The subsectors of some effected sectors due to pandemic COVID-19 have been mentioned in Figs. 25 and 26.

It is also observed that many countries over the world are facing many challenges in the above-mentioned sectors due to COVID-19 situations. Some of the countries effected are listed as follows: Afghanistan, Albania, Algeria, Angola, Argentina, Armenia, Australia, Austria, Azerbaijan, Bangladesh, Belarus, Belgium, Bolivia, Bosnia and

\section{No. of authors contributed in Africa Latin America}

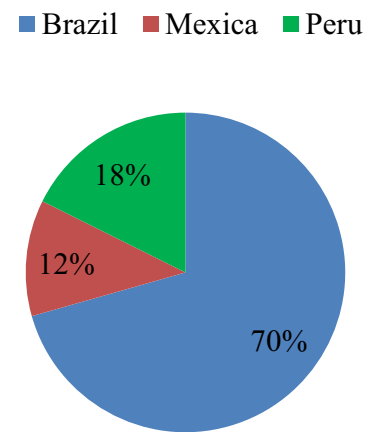

Fig. $21 \%$ of ratio of no. of author's contribution for COVID-related articles using ML, DL \& Others in Latin America 


\section{No of authors contributed in Australia}

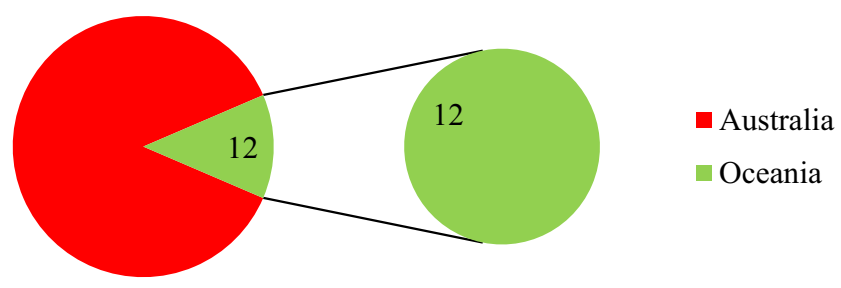

Fig. 22 Usage levels of no. of author's contribution for COVID-related articles using ML, DL \& Others in Australia

Herzegovina, Botswana, Brazil, Bulgaria, Burundi, Cambodia, Cameroon, Canada, Chad, Chile, China, Colombia, Congo, Costa Rica, Croatia, Cuba, Cyprus, Czech Republic, Denmark, Ecuador, Egypt, Estonia, Ethiopia, EU \& Euro-Zone, Finland, France, Georgia, Germany, Ghana, Greece, Greenland, Guatemala, Hong Kong, Hungary, Iceland, India, Indonesia, Iran, Iraq, Ireland, Israel, Italy, Japan, Jordan, Kazakhstan, Kenya, Kosovo, Kuwait, Latvia, Lebanon, Liberia, Libya, Liechtenstein, Lithuania, Luxembourg, Macedonia, Madagascar, Malaysia, Mali, Malta, Mexico, Monaco, Morocco, Mozambique, Namibia, Nepal, Netherlands, New Zealand, Nicaragua, Niger, Nigeria, North Korea, Norway, Other, Pakistan, Panama, Paraguay, Peru, Philippines, Poland, Portugal, Puerto Rico, Qatar, Romania, Russia, Rwanda, Saudi Arabia, Senegal, Serbia, Sierra Leone, Singapore, Slovakia, Slovenia, Somalia, South Africa, South Korea, Spain, Sudan, Sweden, Switzerland, Syria, Taiwan, Tanzania, Thailand, Togo, Tunisia, Turkey, Uganda, UK, Great Britain, Ukraine, United Arab Emirates, Uruguay, USA, Uzbekistan, Venezuela, Vietnam, West Bank and Gaza, Yemen, Zambia, Zimbabwe. These countries are facing many problems like unemployment, transportation, automobile industry, agriculture, exports, health, business of road side vendors etc.

\section{No. of authors contributed in Africa}
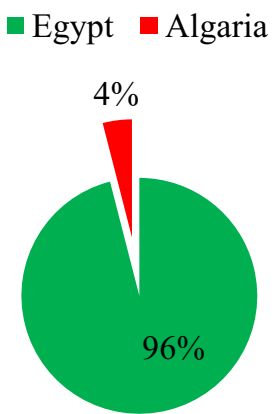

Fig. 24 Usage levels of no of author's contribution for COVID-related articles using ML, DL \& Others in Africa

\section{Discussions}

The overall pandemic of the COVID-19 has become the main countrywide safety issue of several nations. The development of correct prediction models for the epidemic is necessary to offer insights into the consequences and widen of this contagious disease. This paper focuses on an analysis of various intelligent methods in COVID-19 prognosis. Up to now ML and DL methods have shown their efficiency for better understanding the pattern of viral spread. The advanced features of both ML and DL have improved diagnostic accuracy and speed, extended new efficient therapeutic advances, and recognized the majority susceptible citizens potentially with the physiological characteristics [123]. The COVID-19 patient's clinical information along with health conditions has examined with $\mathrm{ML}$ and $\mathrm{DL}$ techniques to not only recognizing any consistent features for the risk assessment, but also the classification of risk as well as forecasting the balanced training of current disease handling and COVID-19 defense. An efficient therapeutic approach is required immediately to treat fast raising COVID-19 patients globally. Due to no useful drug to
Fig. $23 \%$ Usage levels of no. of author's contribution for COVIDrelated articles using ML, DL \& Others in North America

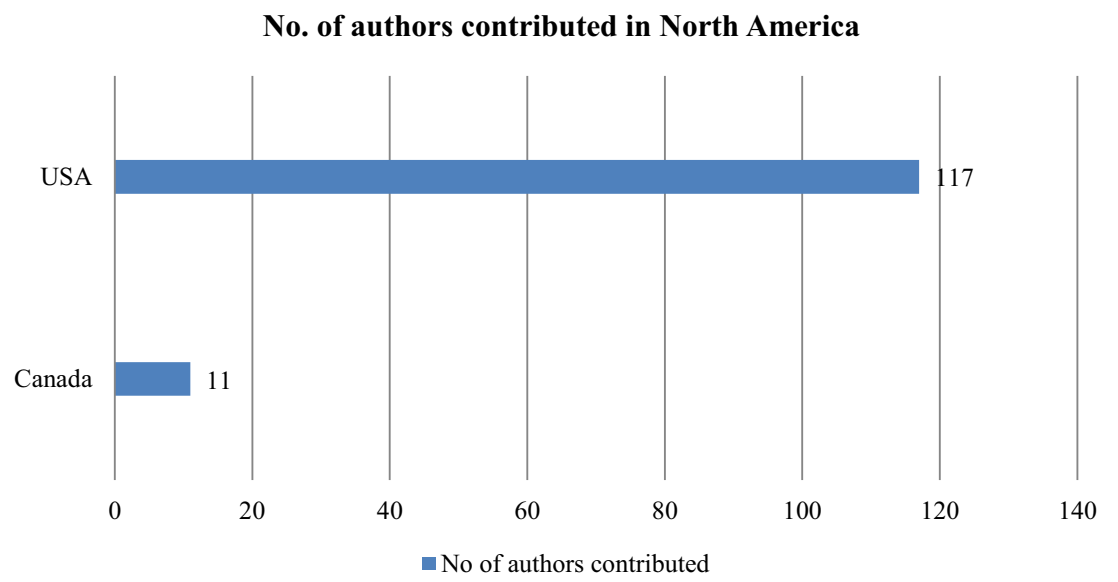


Table 4 Existing surveys comparison with this present study

\begin{tabular}{|c|c|c|c|c|c|}
\hline S.No & Critically reviewed & Intelligent Method Considered & Type of data Observed & $\begin{array}{l}\text { Followed systematic } \\
\text { procedure for literature }\end{array}$ & Ref \\
\hline 1. & No & Only Machine Learning & Image & - & [118] \\
\hline 2. & Yes & Only Machine Learning & Text & $*$ & [119] \\
\hline 3. & No & Only Machine Learning & Text & - & {$[120]$} \\
\hline 4. & Yes & Only Machine Learning & - & $*$ & {$[121]$} \\
\hline 5. & No & Only Machine Learning & Text & - & [122] \\
\hline 6. & Yes & Both Machine Learning and Deep Learning & Image, text and others & $*$ & This Survey \\
\hline
\end{tabular}

* indicates followed systematic procedure for literature

treat patients, it turns serious to build up an efficient enhancement to design or repurpose new clinically certified drug against COVID-19. At this time, a ML-based relocating structure was enhanced to prioritize accessible drugs against COVID-19 for medical trials [111]. Also, a DL based drug detection pipeline has been applied to intend and produce new drug-like compounds against COVID-19 [65] respectively. At present, many attempts [124][125] have been made to enhance new analytical advances with both ML as well as DL. Some of the examples include: ML-based transmission of SARS-CoV-2 analyzing designs utilizing a CRISPR-based virus recognition system was confirmed with high sensitivity and speed [27]. Likewise, due to COVID-19 out broke in a short gap, many researchers have made their study in detection and prediction of COVID-19 by using advanced ML as well as DL techniques such as taxonomic categorization of

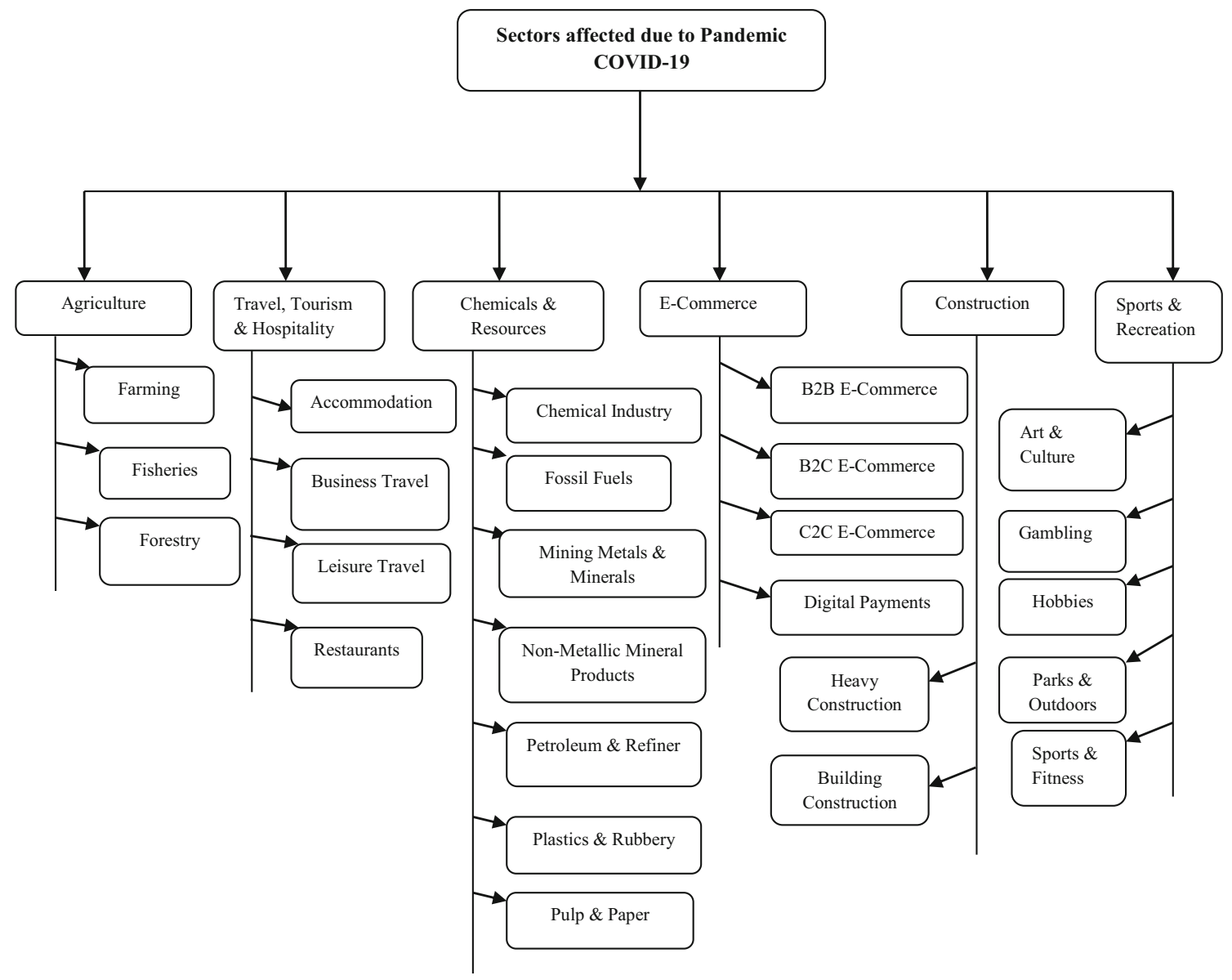

Fig. 25 List of sector and its related subsectors affected due to COVID-19 


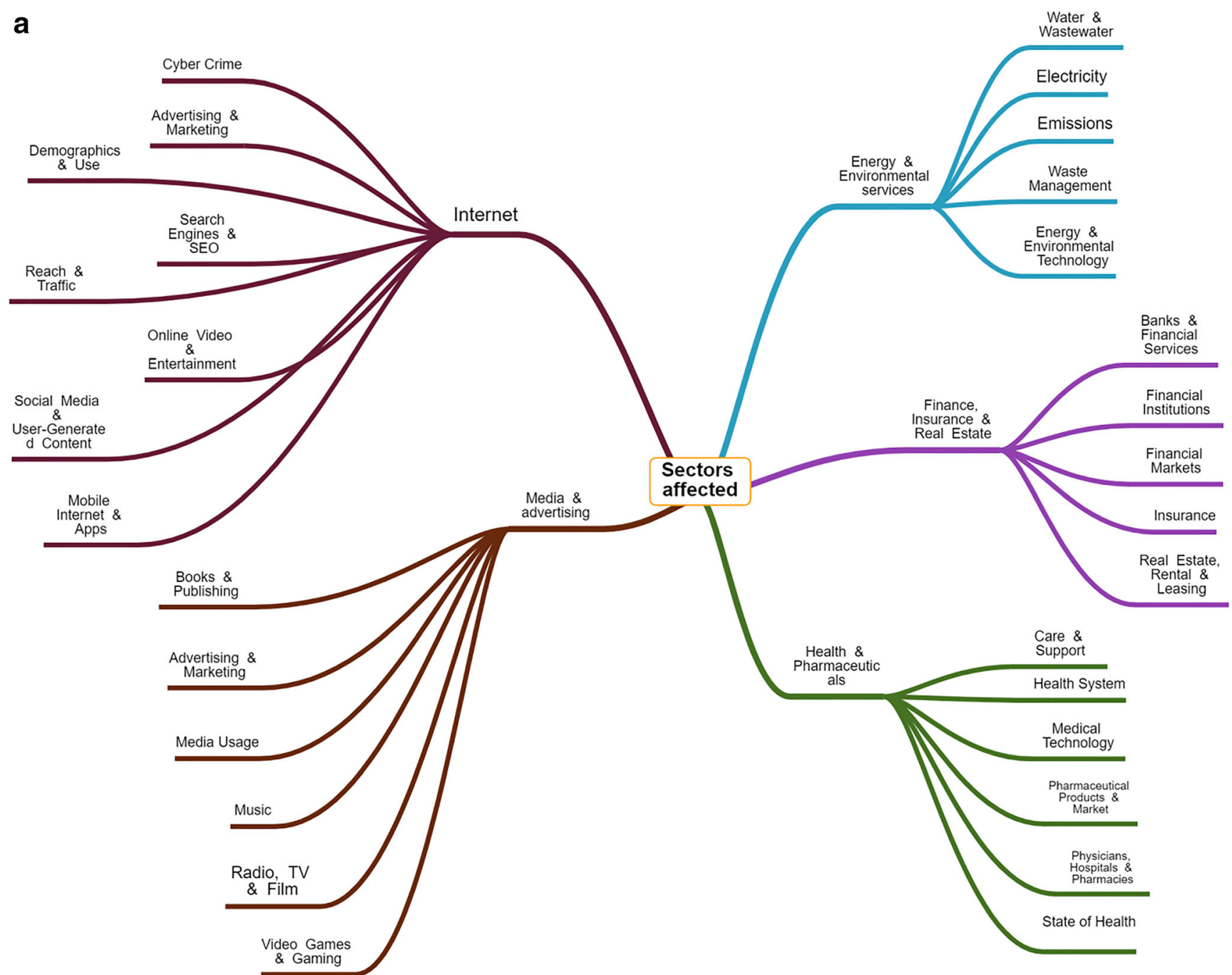

Fig. 26 a List of different Sectors affected by COVID-19. b Other Sectors along with its sub-sectors affected

COVID-19 genomes [16] etc., up-to-now research is mostly limited to detection as well as identification, but many other fields are there to notice and make a further study.

Since the outbreak of this pandemic, several doctors as well as researchers throughout the globe have improved many techniques to predict the COVID-19 in different areas. But the majority of the techniques are failing due to lack of exact solutions that can predict the COVID-19. Each model uses several techniques, builds various statements, generates several results, and fights with uncertainty in numerous ways. The information about tendencies in COVID-19 all over the world consists of requirements of hospitals, the pressure of social distancing, infected, recovered, as well as upcoming cases, and many more. Besides, there was no such recognized liability in an era when the suitability of techniques is important. The majority of the statisticians revealed that the techniques of prediction that are envisaging the number of deaths due to COVID-19 related with certainty were not constant. All the predicting techniques have revealed a wide range of variations in predictions due to a deficiency of accurate information. Likewise, prediction of approaching results based on data of definite cases is an additional cause behind the differences of prediction. Apart from a scattering of the deadly disease, social distancing, demographics, many other factors that include disabilities, chronic disease, low immunity, etc. are need to be concentrate. The major drawback of researchers is facing failures for predicting pandemic in getting exact outcomes. So, to generate more prediction, an additional study has to be passed on improving methods and tools of prediction on most of the biological information. The rise of pandemic COVID-19 is growing day-by-day disregarding various techniques, precautions, and various research articles. The overall confirmed cases have reached to $32,118,316$ along with 23,696,851 recoveries, and 982,360 deaths over the world as per September 24, 2020. Most of the cases, especially in India are calculating to continue growing as procedures of social 
b

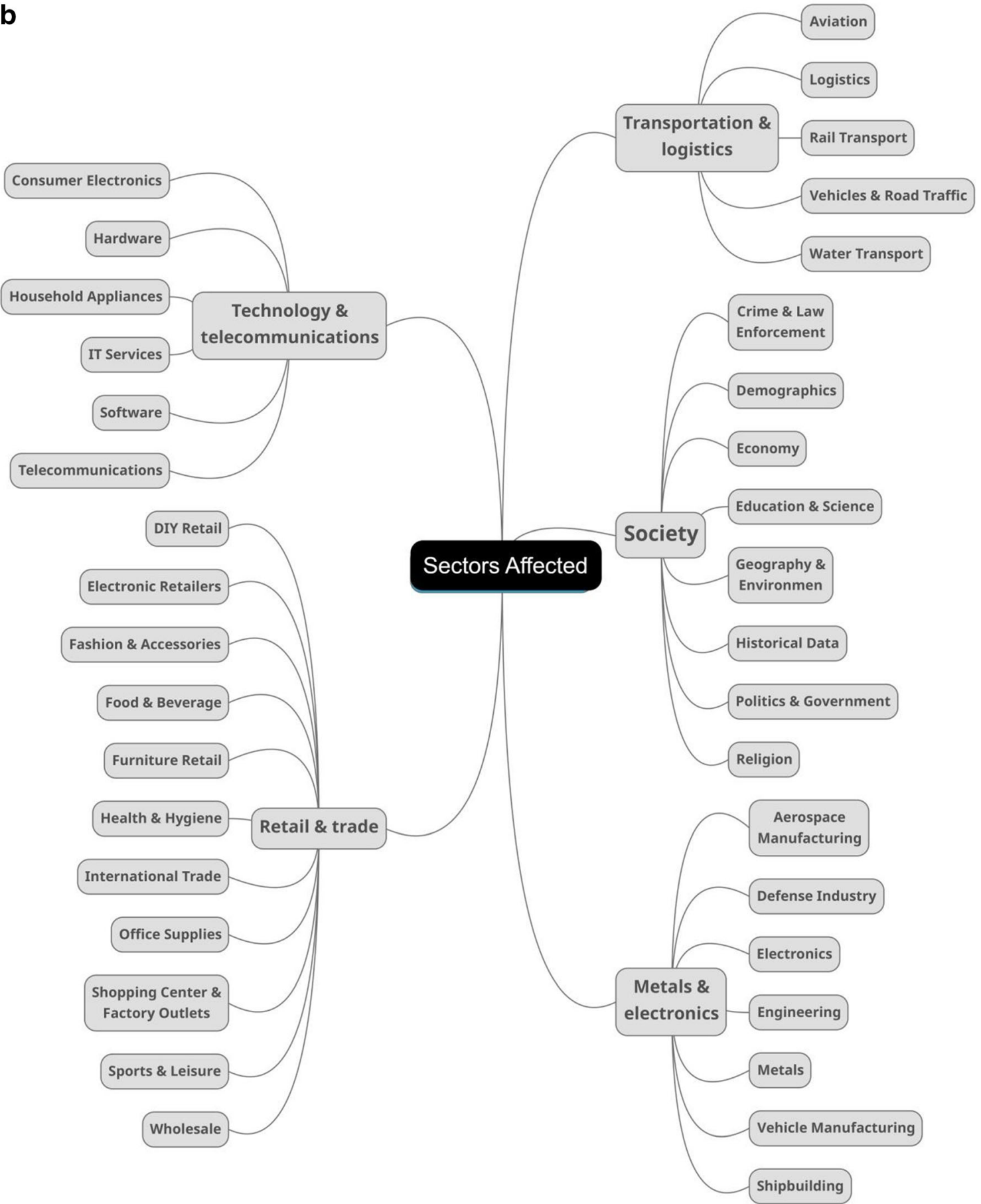

Fig. 26 (continued)

distancing are being calm before deeming a consistent rejection in new cases. Various prediction techniques, solutions, as well as precautions taken by every individual can stop the pandemic spread temporarily and thus aids in the containment of COVID-19. No one has clearness about the conclusion of this epidemic. This deadly disease is incomparable in the alignment of its effortless transmissibility, an assortment of symptoms leaving from one to another in a toxic approach, and the rank of the epidemic has distressed the globe. The stoppage of this pandemic may occur because the citizens grow tired of panic manner and are trained to live with an illness, as the past outbreaks do present ideas of the future. Many deadly diseases include Ebola, smallpox, influenzas, and many more were faced by humans. But all these diseases 
have ended after various deaths because of the execution of quarantines, policy measures, and a few diseases that were stopped after developing vaccines. The methods of the early plagues that came to an end have obtained guidelines to a globe observing for ways to modernize strength and numerous sense of normalcy. Projections behind the continuations of COVID-19 will be temporary, but the stopping of it will engage an accord of the entire thing that guarantees early diseases such as social protective measures, new medicines to lessen symptoms and a vaccine. Besides, several researchers have to work on various confronts to improve medications. So, vaccines are the permanent resolution for ending this epidemic that may need months or even years to be complete. If the medicine is released throughout the world, then pandemic is likely to become extensive to the individuals who are not ill or improved currently.

The grouping of vaccinations and natural exemptions will protect the majority of the population. So, as per our observations, we can be assured that this space can be naturally field by recognizing medicines by using a collection of existing infection medicines like sars- 1 , Spanish flu, and many more with the help of ML and DL methods. Our current study has shown the significance of ML as well as DL for COVID-19. We have majorly focused on the detailed analysis of ML and DL techniques for COVID-19 along with their applications. Based on our study, we noticed that some drawbacks of ML such as deficiency of recognizing equipment, usage of biomedical data, heterogeneity, and fewer medical equipment, etc. made DL techniques as powerful as well as suitable for predicting COVID-19 pandemic. Techniques of DL are suitable for COVID-19 prediction, as these methods can improve health systems to identify the infections. The techniques of DL have outperformed several investigations with loyal data on enhancing the efficacy of medical checks by predicting side effects as well as creative ways. DL is suitable for practicing various layers of nonlinear information and get accurate decisions for a complex problem like a consequence of medical data. The majority of DL characteristics such as recognition, deficiency of human involvement, and better performance made it a more effective and admired technique when compared to ML for COVID-19 prediction.

\section{Conclusion}

From the research analysis conducted in this paper, it is observed that mostly ML and DL have been used for detecting, predicting, identifying as well as the screening of COVID-19. It is also noticed that ML algorithms such as SVM, LR, RF, and KNN have been used for solving problems of COVID-19 from December 2019 to the present day. Still, there is a need to incorporated advanced ML algorithms such as ensemble methods such as bagging, boosting, stacking, etc. Also some other ML methods such as Bayesian networks like Gaussian Naive Bayes, Decision tree algorithms like C4.0, clustering algorithms like K-Means, K-Medians and Hierarchical need to be applied with COVID-19 data for successful detection and classification of different symptoms. Also, it is noticeable that ANNs such Perceptron, back propagation, Radial basis function-based NN, need to be applied with COVID-19 related data. In the case of DL, CNN and its variants have been successfully utilized for resolving COVID-19 issues. Also, higher-order neural networks such as Pi-Sigma NN, Functional link NN, Probabilistic NN, Recurrent NN, Wavelet NN, Petri net NN, Spiking NN, Hopfield NN, Binary NN, Orthogonal NN, Adaptive NN, Polynomial NN and Granular NN, etc. have not yet utilized for COVID-19 data and needs to be applied for better performance analysis. Moreover, other ML approaches such as optimization methods, fuzzy systems etc. may play a crucial role for COVID-19 prognosis in near future.

It is also worth able to mention that every researcher, practitioners as well as others throughout the world are now facing an issue of lack of data. Due to this data lacking, they are not able to apply such kind of methods mentioned above as not all the techniques fit for every data related to COVID-19 that is available now. More real-world datasets with updated COVID-19 related data need to be created, so that it may fulfill the lacking issue. Despite observing clear risks in both the global and Indian economy, there is still a hope that this horrible situation can be evaded. Governments have found out from earlier disasters that the results of a demand obsessed decline can be opposed to government expenditure. Accordingly, many governments are raising their stipulation of financial welfare to society, and guaranteeing business can access to the finances desired to maintain their workers employed during the pandemic. Besides, the exact nature of this disaster denotes that a few sectors like health care, food retail, and e-commerce may have benefits of offering at least some financial expansion to compensate for the damage. In this way, several areas have impacted due to this pandemic and require having research on these sectors also. This study presents a deep review of the usage of ML and DL techniques for COVID-19. The major reason behind this review was to study the experience of this pandemic whole over the world and the main inspiration behind the usage of ML and DL techniques to COVID-19. We mentioned all the ML and DL models that are utilized in COVID-19 along with the efficient incentives. Similarly, the requirement of further research on other sectors except prediction and identifications were also mentioned above accordingly. On the basis of this research work, we strongly recommend that many other researches need to be conducted with ML as well as DL on COVID-19 data with those methods not have applied yet. Also, there is a need to address the challenges of lack of data of COVID-19 to conduct further advanced researches. 
Several sectors had a serious impact on them due to COVID-19. Various COVID-19 cases have been identified in more than 200 countries. Many festivals, gaming events, tournaments, and exhibitions have postponed or canceled. The epidemic has a serious impact on the educational field throughout the world and led to the shutting of schools as well as universities. Aviation, retail, financials, automobiles, and real estate are the five major sectors that are burning in severe pain now. The COVID-19 had a major impact on the global economy. These are extremely unsure times for the economic markets, with nations around the world anguish the threatening effects of the epidemic. No company is resistant to the challenges, and the consequences of the health disaster are anticipated to experience for many more months. The economic damage caused by pandemic has seen in highly effected industries like travel and tourism. To decrease the virus spread, countries restricted from traveling. This decrease in consumer demand causes airlines to drop planned revenue. The same scenario has noticed in many other industries. Likewise, the impact of COVID-19 in the Indian economy has remarked in various sectors. The serious trade impact of COVID-19 for India is likely to be $\$ 348$ million. The union of Indian Industry put forward the impacts for various domains includes auto, pharma, chemicals, electronics, solar power, information technology, shipping, textiles, tourism, and aviation, etc. where the stoppage in china has forbidden imports of several components distressing Indian auto component industry as well as auto manufacturers. Even though India is placed at the top for drug exporting, the domestic pharma trade relies greatly on the importation of bulk drugs. But the outbreak of COVID-19 has stopped this importing as well as exporting process. Local dyestuff in India are completely relying on import of various raw equipment from china including chemicals, but late shipment from china are distressing the colorant and colorant stuff industries in India. China was the main supplier of products that are utilized in electronic industries, but due to this pandemic, the electronic industry in India is facing supply disturbance and production falling. IT companies completely depend on humans and are unable to work because of constraints in the movement of the public started from lockdown and quarantine concerns. In this way, the Indian economy had faced several pitfalls and started facing many shortfalls.

\section{Compliance with Ethical Standards}

I on behalf of the authors would like to state that the above manuscript is our original research work and it has not been published elsewhere. Also, it has not been submitted to any journal for publication.

Conflict of interest The authors declare that this manuscript has no conflict of interest with any other published source and has not been published previously (partly or in full). No data have been fabricated or manipulated to support our conclusions.

\section{References}

1. McMichael AJ (2004) Environmental and social influences on emerging infectious diseases: past, present and future. Philos Trans R Soc Lond Ser B Biol Sci 359(1447):1049-1058

2. Paules CI, Marston HD, Fauci AS (2020) Corona virus infectionsmore than just the common cold. JAMA 323(8):707-708

3. Estola T (1970) Corona viruses, a new group of animal RNA viruses. Avian Dis. 14(2):330-336

4. World Health Organization (2020) WHO novel corona virusThailand (ex-China). Geneva, Swiss. Available: http://www.who. int/csr/don/14-january-2020-novel-coronavirusthailand/en/. Accessed 14 July 2020

5. Lu H, Stratton CW, Tang YW (2020) Outbreak of pneumonia of unknown etiology in Wuhan China: the mystery and the miracle. J Med Virol 92(4):401-402

6. Kwok KO, Tang A, Wei VW, Park WH, Yeoh EK, Riley S (2019) Epidemic models of contact tracing: Systematic review of transmission studies of severe acute respiratory syndrome and middle east respiratory syndrome. Comput Struct Biotechnol J 17:186-194

7. Debnath S, Barnaby DP, Coppa K, Makhnevich A, Kim EJ, Chatterjee S, Hirsch JS (2020) Machine learning to assist clinical decision-making during the COVID-19 pandemic. Bioelectronic Med 6(1):1-8

8. van der Schaar M, Alaa A (2020) How artificial intelligence and machine learning can help healthcare systems respond to COVID19. https://www.vanderschaar-lab.com/NewWebsite/covid-19/ post1/paper.pdf

9. Altan A, Karasu S (2020) Recognition of COVID-19 disease from X-ray images by hybrid model consisting of 2D curvelet transform, chaotic salp swarm algorithm and deep learning technique. Chaos, Solitons Fractals 140:110071. https://www.sciencedirect.com/ science/article/pii/S0960077920304689

10. KayvanJoo AH, Ebrahimi M, Haqshenas G (2014) Prediction of hepatitis $C$ virus interferon/ribavirin therapy outcome based on viral nucleotide attributes using machine learning algorithms. BMC Res Notes 7(1):565

11. Klon AE, Glick M, Davies JW (2004) Application of machine learning to improve the results of high-throughput docking against the HIV-1 protease. J Chem Inf Comput Sci 44(6):2216-2224

12. Ekins S, Freundlich JS, Clark AM, Anantpadma M, Davey RA, Madrid P (2015) Machine learning models identify molecules active against the Ebola virus in vitro. F1000Res 4:1091

13. Bengio Y, Courville A, Vincent P (2013) Representation learning: A review and new perspectives. IEEE Trans Pattern Anal Mach Intell 35(8):1798-1828

14. Lundervold AS, Lundervold A (2019) An overview of deep learning in medical imaging focusing on MRI. Zeitschrift für Medizinische Physik 29(2):102-127

15. Razzak MI, Naz S, Zaib A (2018) Deep learning for medical image processing: Overview, challenges and the future. In: Classification in BioApps. Springer, Cham, pp 323-350

16. Randhawa GS, Soltysiak MP, Roz E, de Souza H, Hill CP, K. A., \& Kari L, (2020) Machine learning using intrinsic genomic signatures for rapid classification of novel pathogens: COVID-19 case study. PLoS One 15(4):e0232391

17. Apostolopoulos ID, Mpesiana TA (2020) COVID-19: automatic detection from x-ray images utilizing transfer learning with convolutional neural networks. Phys Eng Sci Med 43(2):635-640

18. Cohen JP (2020)COVID-19 image data collection. https://github. com/ieee8023/COVID-chestxray-dataset. Accessed 21 November 2020

19. Kaggle (2020)https://www.kaggle.com/andrewmvd/convid19-Xrays. Accessed 21 November 2020 
20. Kermany DS, Goldbaum M, Cai W et al (2018) Identifying medical diagnoses and treatable diseases by image-based deep learning. Cell 172:1122-1131.e9. https://doi.org/10.1016/j.cell.2018.02.010

21. Kang H, Xia L, Yan F, Wan Z, Shi F, Yuan H, Jiang H, Wu D, Sui H, Zhang C, Shen D (2020) Diagnosis of Coronavirus Disease 2019 (COVID-19) with structured latent multi-view representation learning. IEEE Trans Med Imaging 39(8):2606-2614. https://doi.org/10. 1109/TMI.2020.2992546

22. Cheng FY, Joshi H, Tandon P, Freeman R, Reich DL, Mazumdar $\mathrm{M}$, Kia A (2020) Using machine learning to predict ICU transfer in hospitalized COVID-19 patients. J Clin Med 9(6):1668

23. Khanday AMUD, Rabani ST, Khan QR, Rouf N, Din MMU (2020) Machine learning based approaches for detecting COVID-19 using clinical text data. Int J Inf Technol 12:731-739. https://doi.org/10. 1007/s41870-020-00495-9

24. Assaf D, Gutman YA, Neuman Y, Segal G, Amit S, Gefen-Halevi S, Shilo N, Epstein A, Mor-Cohen R, Biber A, Rahav G, Itzchak L, Tirosh A (2020) Utilization of machine-learning models to accurately predict the risk for critical COVID-19. Int Emerg Med 15: 1435-1443. https://doi.org/10.1007/s11739-020-02475-0

25. Rustam F, Reshi AA, Mehmood A, Ullah S, On B, Aslam W, Choi GS (2020)COVID-19 future forecasting using supervised machine learning models. IEEE Access 8:101489-101499. https://doi.org/ 10.1109/ACCESS.2020.2997311

26. Sharma S (2020) Drawing insights from COVID-19-infected patients using CT scan images and machine learning techniques: a study on 200 patients. Environ Sci Pollut Res 27:37115-37163. https://doi.org/10.1007/s11356-020-10133-3

27. Peng Y, Nagata MH (2020) An empirical overview of nonlinearity and overfitting in machine learning using COVID-19 data. Chaos, Solitons Fractals 139:110055

28. Ardabili SF, Mosavi A, Ghamisi P, Ferdinand F, Varkonyi-Koczy AR, Reuter U, Rabczuk T, Atkinson PM (2020)COVID-19 outbreak prediction with machine learning. Available at SSRN 3580188

29. Nemati M, Ansary J, Nemati N (2020) Machine learning approaches in COVID-19 survival analysis and discharge time likelihood prediction using clinical data. Patterns 1:100074

30. Sun CLF, Zuccarelli E, Zerhouni EGA, Lee J, Muller J, Scott KM, Lujan AM, Levi R (2020) Predicting COVID-19 infection risk and related risk drivers in nursing homes: a machine learning approach. J Am Med Dir Assoc 21(11):1533-1538.e6. https://doi.org/10. 1016/j.jamda.2020.08.030

31. Burdick H, Lam C, Mataraso S, Siefkas A, Braden G, McCoy A, Vincen JL, Green-Saxena A, Barnes G, Hoffman J, Calvert J, Pellegrini E, Das R (2020) Prediction of respiratory decompensation in Covid-19 patients using machine learning: The READY trial. Comput Biol Med 124:103949. https://doi.org/10.1016/j. compbiomed.2020.103949

32. Kavadi DP, Patan R, Ramachandran M, Gandomi AH (2020) Partial derivative nonlinear global pandemic machine learning prediction of covid 19. Chaos, Solitons Fractals 139:110056

33. Banerjee A, Ray S, Vorselaars B, Kitson J, Mamalakis M, Weeks S, Mackenzie LS (2020) Use of machine learning and artificial intelligence to predict SARS-CoV-2 infection from full blood counts in a population. Int Immunopharmacol 86:106705

34. Wang P, Zheng X, Li J, Zhu B (2020) Prediction of epidemic trends in COVID-19 with logistic model and machine learning technics. Chaos, Solitons Fractals 139:110058

35. Han Z, Wei B, Hong Y, Li T, Cong J, Zhu X, Zhang W (2020) Accurate screening of COVID-19 using attention based deep 3D multiple instance learning. IEEE Trans Med Imaging 39(8):2584 2594

36. Vaid S, Cakan C, Bhandari M (2020) Using machine learning to estimate unobserved COVID-19 infections in North America. J
Bone Joint Surg Am 102(13):e70. https://doi.org/10.2106/JBJS. 20.00715

37. Elaziz MA, Hosny KM, Salah A, Darwish MM, Lu S, Sahlol AT (2020) New machine learning method for image-based diagnosis of COVID-19. PLoS One 15(6):e0235187

38. Ahamad MM, Aktar S, Rashed-Al-Mahfuz M, Uddin S, Liò P, Xu H, ... Moni MA (2020) A machine learning model to identify early stage symptoms of SARS-Cov-2 infected patients. Expert Syst Appl 160:113661

39. Brinati D, Campagner A, Ferrari D, Locatelli M, Banfi G, Cabitza F (2020) Detection of COVID-19 infection from routine blood exams with machine learning: a feasibility study. J Med Syst 44:135. https://doi.org/10.1007/s10916-020-01597-4

40. Hasan N (2020) A methodological approach for predicting COVID-19 epidemic using EEMD-ANN hybrid model. Internet Things 11:100228

41. Farid AA, Selim GI, Khater HAA (2020) A novel approach of CT images feature analysis and prediction to screen for corona virus disease (COVID-19). Int J Sci Eng Res 11(3):1-9

42. Shaban WM, Rabie AH, Saleh AI, Abo-Elsoud MA (2020) A new COVID-19 Patients Detection Strategy (CPDS) based on hybrid feature selection and enhanced KNN classifier. Knowl-Based Syst 205:106270

43. Ou S, He X, Ji W, Chen W, Sui L, Gan Y, Lu Z, Lin Z, Deng S, Przesmitzki S, Bouchard J (2020) Machine learning model to project the impact of COVID-19 on US motor gasoline demand. Nat Energy 5:666-673. https://doi.org/10.1038/s41560-020-0662-1

44. Samuel J, Ali GG, Rahman M, Esawi E, Samuel Y (2020)Covid-19 public sentiment insights and machine learning for tweets classification. Information 11(6):314

45. Pinter G, Felde I, Mosavi A, Ghamisi P, Gloaguen R (2020)COVID-19 pandemic prediction for hungary; a hybrid machine learning approach. Mathematics 8(6):890

46. Carrillo-Larco RM, Castillo-Cara M (2020) Using country-level variables to classify countries according to the number of confirmed COVID-19 cases: an unsupervised machine learning approach. Wel lcome Open Res 5(56):56

47. Benitez-Pena S, Carrizosa E, Guerrero V, Dolores M (2020)Shortterm predictions of the evolution of COVID-19 in Andalusia. An ensemble method. https://www.researchgate.net/publication/ 340716304_Short-Term Predictions of the Evolution of COVID-19 in Andalusia An Ensemble Method. Accessed $2 \overline{1}$ November 2020

48. Zhong L, Mu L, Li J, Wang J, Yin Z, Liu D (2020) Early prediction of the 2019 novel corona virus outbreak in the mainland China based on simple mathematical model. IEEE Access 8:51761-51769

49. Yadav M, Perumal M, Srinivas M (2020) Analysis on novel coronavirus (covid-19) using machine learning methods. Chaos, Solitons Fractals 139:110050

50. Singh D, Kumar V, Kaur M (2020) Classification of COVID-19 patients from chest $\mathrm{CT}$ images using multi-objective differential evolution-based convolutional neural networks. Eur J Clin Microbiol Infect Dis :1-11

51. Khan AI, Shah JL, Bhat MM (2020) Coronet: A deep neural network for detection and diagnosis of COVID-19 from chest x-ray images. Comput Methods Prog Biomed 196:105581

52. Sethy PK, Behera SK (2020) Detection of corona virus Disease (COVID-19) based on Deep Features. Preprints: 2020030300. https://doi.org/10.20944/preprints202003.0300.v1

53. Alakus TB, Turkoglu I (2020) Comparison of deep learning approaches to predict COVID-19 infection. Chaos, Solitons Fractals 140:110120

54. Zeng X, Song X, Ma T, Pan X, Zhou Y, Hou Y, Zhang Z, Li K, Karypis G, Cheng F (2020) Repurpose open data to discover therapeutics for COVID-19 using deep learning. J Proteome Res 19(11):4624 4636. https://doi.org/10.1021/acs.jproteome.0c00316 
55. Yoo SH, Geng H, Chiu TL, Yu SK, Cho DC, Heo J, Min BJ (2020) Deep learning-based decision-tree classifier for COVID-19 diagnosis from chest $\mathrm{x}$-ray imaging. Front Med 7:427

56. Sedik A, Iliyasu AM, El-Rahiem A, Abdel Samea ME, AbdelRaheem A, Hammad M, El-Latif AAA (2020) Deploying machine and deep learning models for efficient data-augmented detection of COVID-19 infections. Viruses 12(7):769

57. Nour M, Cömert Z, Polat K (2020) A novel medical diagnosis model for COVID-19 infection detection based on deep features and bayesian optimization. Appl Soft Comput 97(Part A):106580

58. Jamshidi M, Lalbakhsh A, Talla J, Peroutka Z, Hadjilooei F, Lalbakhsh, P.,... Sabet, A. (2020) Artificial intelligence and COVID-19: deep learning approaches for diagnosis and treatment. IEEE Access 8:109581-109595

59. Waheed A, Goyal M, Gupta D, Khanna A, Al-Turjman F, Pinheiro PR (2020) COVIDgan: Data augmentation using auxiliary classifier gan for improved COVID-19 detection. IEEE Access 8:9191691923 (IEEE COVID)

60. Chest X-ray dataset. Available: https://github.com/ieee8023/ COVID-chestxray-dataset. Accessed 11 November 2020

61. COVID19 Radiography Database. Available: https://www.kaggle. com/tawsifurrahman/COVID19-radiography-database. Accessed 20 November 2020

62. COVID-19 Chest X-ray Dataset Initiative. Available: https:// github.com/agchung/Figure1-COVID-chestxray-dataset. Accessed 21 November 2020

63. Chimmula VKR, Zhang L (2020) Time series forecasting of COVID-19 transmission in Canada using LSTM networks. Chaos, Solitons Fractals 135:109864

64. Uddin MI, Shah SAA, Al-Khasawneh MA (2020) A novel deep convolutional neural network model to monitor people following guidelines to avoid COVID-19. J Sensor 2020:1-15

65. Zhavoronkov A et al (2020) Potential COVID-2019 3C-like protease inhibitors designed using generative deep learning approaches. Insilico Medicine Hong Kong Ltd A, 307 E1

66. Zeroual A, Harrou F, Dairi A, Sun Y (2020) Deep learning methods for forecasting COVID-19 time-Series data: A Comparative study. Chaos, Solitons Fractals 140:110121

67. Huang L, Han R, Ai T, Yu P, Kang H, Tao Q, Xia L (2020) Serial quantitative chest ct assessment of COVID-19: deep-learning approach. Radiology: Cardiothoracic Imaging 2(2):e200075

68. Farooq J, Bazaz MA (2020) A novel adaptive deep learning model of Covid-19 with focus on mortality reduction strategies. Chaos, Solitons Fractals 138:110148

69. Loey M, Smarandache F, Khalifa M (2020) Within the lack of chest COVID-19 X-ray dataset: a novel detection model based on GAN and deep transfer learning. Symmetry 12(4):651

70. Pathak Y, Shukla PK, Tiwari A, Stalin S, Singh S, Shukla PK (2020) Deep transfer learning based classification model for COVID-19 disease. IRBM, Elsevier, Amsterdam

71. Arora P, Kumar H, Panigrahi BK (2020) Prediction and analysis of COVID-19 positive cases using deep learning models: A descriptive case study of India. Chaos, Solitons Fractals 139:110017

72. Shi W et al (2020a) Deep learning-based quantitative computed tomography model in predicting the severity of COVID-19: a retrospective study in 196 patients. https://doi.org/10.2139/ssrn. 3546089

73. Cao Y, Xu Z, Feng J, Jin C, Han X, Wu H, Shi H (2020) Longitudinal assessment of COVID-19 using a deep learningbased quantitative ct pipeline: Illustration of two cases. Radiology: Cardiothoracic Imaging 2(2):e200082

74. Brunese L, Mercaldo F, Reginelli A, Santone A (2020) Explainable deep learning for pulmonary disease and coronavirus COVID-19 detection from X-rays. Comput Methods Programs Biomed 196: 105608
75. Civit-Masot J, Luna-Perejón F, Domínguez Morales M, Civit A (2020) Deep learning system for COVID-19 diagnosis aid using X-ray pulmonary images. Appl Sci 10(13):4640

76. Sethi R, Mehrotra M, Sethi D (2020) Deep learning based diagnosis recommendation for COVID-19 using chest x-rays images. In: 2020 Second International Conference on Inventive Research in Computing Applications (ICIRCA). IEEE, New York, pp 1-4

77. Jain G, Mittal D, Thakur D, Mittal MK (2020) A deep learning approach to detect Covid-19 coronavirus with X-Ray images. Biocybern Biomed Eng 40(4):1391-1405

78. Qjida M, Ben-fares A, Mechbal Y, Amakdouf H, Maaroufi M, Alami B, Qjidaa H (2020) Development of a clinical decision support system for the early detection of COVID-19 using deep learning based on chest radiographic images. In: International Conference on Intelligent Systems and Computer Vision (ISCV), Fez, Morocco, 2020, pp 1-6. https://doi.org/10.1109/ISCV49265. 2020.9204282

79. Das NN, Kumar N, Kaur M, Kumar V, Singh D (2020) Automated deep transfer learning-based approach for detection of COVID-19 infection in chest X-rays. IRBM. https://doi.org/10.1016/j.irbm. 2020.07.001

80. Ouchicha C, Ammor O, Meknassi M (2020) CVDNet: A novel deep learning architecture for detection of coronavirus (Covid-19) from chest x-ray images. Chaos, Solitons Fractals 140:110245

81. Santosh K, Das D, Pal U (2020) Truncated inception net: COVID19 outbreak screening using chest x-rays, https://doi.org/10.1007/ s13246-020-00888-x

82. Zhou L, Li Z, Zhou J, Li H, Chen Y, Huang Y, Abdelkareem F (2020) A rapid, accurate and machine-agnostic segmentation and quantification method for CT-based COVID-19 diagnosis. IEEE Trans Med Imaging 39(8):2638-2652

83. Ni Q, Sun ZY, Qi L, Chen W, Yang Y, Wang L, Zhang X, Yang L, Fang Y, Xing Z, Zhou Z, Yu Y, Yu GM, Zhang LJ (2020) A deep learning approach to characterize 2019 coronavirus disease (COVID-19) pneumonia in chest CT images. Eur Radiol 30(12): 6517-6527. https://doi.org/10.1007/s00330-020-07044-9

84. Zhang HT, Zhang JS, Zhang HH, Nan YD, Zhao Y, Fu EQ, Xie YH, Liu W, Li WP, Zhang HJ, Jiang H, Li CM, Li YY, Ma RN, Dang SK, Gao BB, Zhang XJ, Zhang T (2020) Automated detection and quantification of COVID-19 pneumonia: CT imaging analysis by a deep learning-based software. Eur J Nucl Med Mol Imaging 47(11):2525-2532. https://doi.org/10.1007/s00259-02004953-1

85. Apostolopoulos ID, Aznaouridis SI, Tzani MA (2020) Extracting possibly representative COVID-19 biomarkers from x-ray images with deep learning approach and image data related to pulmonary diseases. J Med Biol Eng 40:462-469

86. Hu S, Gao Y, Niu Z, Jiang Y, Li L, Xiao X, Ye H (2020) Weakly supervised deep learning for covid-19 infection detection and classification from ct images. IEEE Access 8:118869-118883

87. Arora K, Bist AS, Chaurasia S, Prakash R (2020) Analysis of deep learning techniques for COVID-19 detection. Int J Sci Res Eng Manag (ijsrem) i 4(4):1-5

88. Pereira RM, Bertolini D, Teixeira LO, Silla CN Jr, Costa YM (2020)COVID-19 identification in chest X-ray images on flat and hierarchical classification scenarios. Comput Methods Prog Biomed 194:105532

89. Marques G, Agarwal D, de la T Díez I (2020) Automated medical diagnosis of COVID-19 through EfficientNet convolutional neural network. Appl Soft Comput 96:106691. https://doi.org/10.1016/j. asoc.2020.106691

90. Wang N, Liu H, Xu C (2020) Deep learning for the detection of COVID-19 using transfer learning and model integration. In: 2020 IEEE 10th International Conference on Electronics Information and Emergency Communication (ICEIEC). IEEE, New York, pp 281284 
91. Ayyoubzadeh SM, Ayyoubzadeh SM, Zahedi H, Ahmadi M, Kalhori SRN (2020) Predicting COVID-19 incidence through analysis of google trends data in Iran: Data mining and deep learning pilot study. JMIR Public Health Surveill 6(2):e18828

92. Minaee S, Kafieh R, Sonka M, Yazdani S, Soufi GJ (2020) Deepcovid: Predicting covid-19 from chest x-ray images using deep transfer learning. Med Image Anal 65:101794. https://doi.org/10. 1016/j.media.2020.101794

93. Butt C, Gill J, Chun D, Babu BA (2020) Deep learning system to screen coronavirus disease 2019 pneumonia. Appl Intell. https:// doi.org/10.1007/s10489-020-01714-3

94. Panwar H, Gupta PK, Siddiqui MK, Morales-Menendez R, Singh V (2020) Application of deep learning for fast detection of COVID19 in x-rays using nCOVnet. Chaos, Solitons Fractals 138:109944

95. Ozturk T, Talo M, Yildirim EA, Baloglu UB, Yildirim O, Acharya UR (2020) Automated detection of COVID-19 cases using deep neural networks with X-ray images. Comput Biol Med 121:103792

96. Ardakani AA, Kanafi AR, Acharya UR, Khadem N, Mohammadi A (2020) Application of deep learning technique to manage COVID-19 in routine clinical practice using CT images: Results of 10 convolutional neural networks. Comput Biol Med 121:103795

97. Toğaçar M, Ergen B, Cömert Z (2020)COVID-19 detection using deep learning models to exploit social mimic optimization and structured chest X-ray images using fuzzy color and stacking approaches. Comput Biol Med 121:103805

98. Rahimzadeh M, Attar A (2020) A modified deep convolutional neural network for detecting COVID-19 and pneumonia from chest $\mathrm{X}$-ray images based on the concatenation of Xception and ResNet50V2. Inf Med Unlocked 19:100360

99. Ucar F, Korkmaz D (2020) COVIDiagnosis-Net: Deep BayesSqueezeNet based diagnostic of the Coronavirus disease 2019 (COVID-19) from x-ray images. Med Hypotheses 140:109761

100. Roy S, Menapace W, Oei S, Luijten B, Fini E, Saltori C, Peschiera E (2020) Deep learning for classification and localization of COVID-19 markers in point-of-care lung ultrasound. IEEE Trans Med Imaging 39:2676-2687

101. Wang X, Deng X, Fu Q, Zhou Q, Feng J, Ma H, Zheng C (2020) A weakly-supervised framework for COVID-19 classification and lesion localization from chest CT. IEEE Trans Med Imaging 39(8):2615-2625

102. Fan DP, Zhou T, Ji GP, Zhou Y, Chen G, Fu H, Shao L (2020) InfNet: Automatic COVID-19 lung infection segmentation from CT images. IEEE Trans Med Imaging 39(8):2626-2637

103. Oh Y, Park S, Ye JC (2020) Deep learning COVID-19 features on cxr using limited training data sets. IEEE Trans Med Imaging 39(8):2688-2700

104. Zhang H, Saravanan KM, Yang Y, Hossain MT, Li J, Ren X, Pan Y, Wei Y (2020) Deep learning based drug screening for Novel Coronavirus 2019-nCov. Interdiscip Sci Comput Life Sci 12(3): 368-376. https://doi.org/10.1007/s12539-020-00376-6

105. Rajaraman S, Antani S (2020) Weakly labeled data augmentation for deep learning: a study on COVID-19 detection in chest $\mathrm{x}$-rays. Diagnostics 10(6):358

106. Vaid S, Kalantar R, Bhandari M (2020) Deep learning COVID-19 detection bias: accuracy through artificial intelligence. Int Orthop 44:1539-1542

107. Wu X, Hui H, Niu M, Li L, Wang L, He B, Zha Y (2020) Deep learning-based multi-view fusion model for screening 2019 novel coronavirus pneumonia: a multicentre study. Eur J Radiol 128: 109041

108. El Asnaoui K, Chawki Y (2020) Using X-ray images and deep learning for automated detection of coronavirus disease. J Biomol Struct Dyn 22:1-12. https://doi.org/10.1080/07391102.2020. 1767212
109. CSSEGISandData/COVID-19 (2020) Available: https://www. github.com/CSSEGISandData/COVID-19. Accessed 21 November 2020

110. Tuli S, Tuli S, Tuli R, Gill SS (2020) Predicting the growth and trend of COVID-19 pandemic using machine learning and cloud computing. Internet Things 11:100222

111. Zame WR, Bica I, Shen C, Curth A, Lee HS, Bailey S, Weatherall J, Wright D, Bretz F, van der Schaar M (2020) Machine learning for clinical trials in the era of COVID-19. Stat Biopharm Res 12(4):506-517. https://doi.org/10.1080/ 19466315.2020.1797867

112. Xu B, Kraemer MU, Gutierrez B, Mekaru S, Sewalk K, Loskill A, Li S (2020) Open access epidemiological data from the COVID19 outbreak. Lancet Infect Dis 20(5):534

113. Frazer JS, Shard A, Herdman J (2020) Involvement of the opensource community in combating the worldwide COVID-19 pandemic: a review. J Med Eng Technol 44(4):169-176

114. Yang W, Yan F (2020) Patients with RT-PCR-confirmedCOVID19 and normal chest CT. Radiology 295(2):E3-E3

115. Ai T, Yang Z, Hou H, Zhan C, Chen C, Lv W, Xia L (2020) Correlation of chest CT and RT-PCR testing in coronavirus disease 2019 (COVID-19) in China: a report of 1014 cases. Radiology 296(2):200642

116. Shi F, Wang J, Shi J, Wu Z, Wang Q, Tang Z, Shen D (2020) Review of artificial intelligence techniques in imaging data acquisition, segmentation and diagnosis for COVID-19. IEEE Rev Biomed Eng. https://doi.org/10.1109/RBME.2020.2987975

117. McCall B (2020)COVID-19 and artificial intelligence: protecting health-care workers and curbing the spread. Lancet Digit Health 2(4):e166-e167

118. Lalmuanawma S, Hussain J, Chhakchhuak L (2020) Applications of machine learning and artificial intelligence for Covid-19 (SARS-CoV-2) pandemic: A review. Chaos, Solitons Fractals 139:110059

119. Albahri AS, Hamid RA (2020) Role of biological data mining and machine learning techniques in detecting and diagnosing the Novel Coronavirus (COVID-19): A systematic review. J Med Syst 44(7):122

120. Sujath R, Chatterjee JM, Hassanien AE (2020) A machine learning forecasting model for COVID-19 pandemic in India. Stoch Env Res Risk A 34:959-972

121. Francesca De Felice, PolimenI A (2020) Coronavirus disease (COVID-19): A machine learning bibliometric analysis. In Vivo 34(3 suppl):1613-1617

122. Yadav RS (2020) Data analysis of COVID-2019 epidemic using machine learning methods: a case study of India. Int J Inf Technol 12:1321-1330

123. Alimadadi A, Aryal S, Manandhar I, Munroe PB, Joe B, Cheng X (2020) Artificial intelligence and machine learning to fight COVID-19. https://doi.org/10.1152/physiolgenomics. 00029.2020

124. Catelli R, Gargiulo F, Casola V, De Pietro G, Fujita H, Esposito M (2020) Crosslingual named entity recognition for clinical deidentification applied to a COVID-19 Italian data set. Appl Soft Comput 97:106779

125. Hernandez-Matamoros A, Fujita H, Hayashi T, Perez-Meana H (2020) Forecasting of COVID19 per regions using ARIMA models and polynomial functions. Appl Soft Comput 96: 106610

Publisher's note Springer Nature remains neutral with regard to jurisdictional claims in published maps and institutional affiliations. 


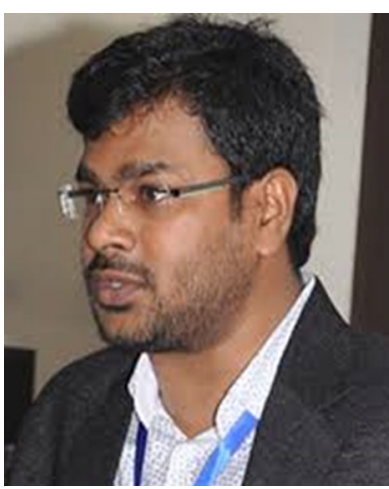

Janmenjoy Nayak (M'19) is working as an Associate Professor, Department of Co m p u e r S c i e n c e and Engineering, Aditya Institute of Technology and Management (AITAM), Tekkali, AP- 532201, India. He has published more than 110 research papers in various reputed peer reviewed Referred Journals, International Conferences and Book Chapters. $\mathrm{He}$ is the recipient of Best researcher award from Jawaharlal Nehru University of Technology, Kakinada, Andhra Pradesh for the AY: 2018-2019 and many others. His area of interest includes data mining, nature inspired algorithms and soft computing. He is the regular member of IEEE and life member of some of the reputed societies like CSI India, etc.He has edited 12 books from various publishers such as Elsevier and Springer. He has been serving as Guest Editor of various journal special issue from Elsevier, Springer and Inderscience.

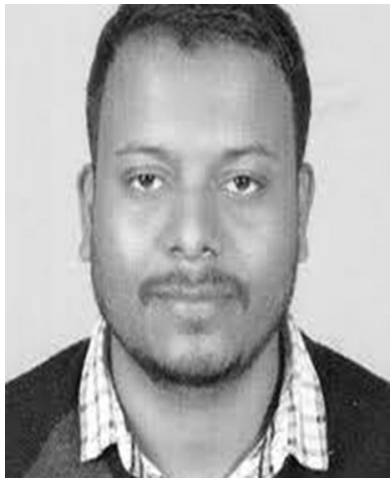

Bighnaraj Naik (M'19) is an Assistant Professor in the Department of Computer Application, Veer SurendraSai University of Technology (Formerly UCE Burla), Odisha, India. He received his $\mathrm{Ph} . \mathrm{D}$. in Computer Science and Engineering, M.Tech. in Computer Science and Engineering and B.E. in information technology in 2016, 2009 and 2006, respectively. He has published more than 90 research papers in various reputed peer reviewed International Journals, Conferences and Book Chapters. He has edited four books from various publishers such as Elsevier, Springer and IGI Global. At present, he has more than ten years of teaching experience in the field of Computer Science and IT. He is a member of IEEE. His area of interest includes Data Mining, Computational Intelligence, Soft Computing and its applications.

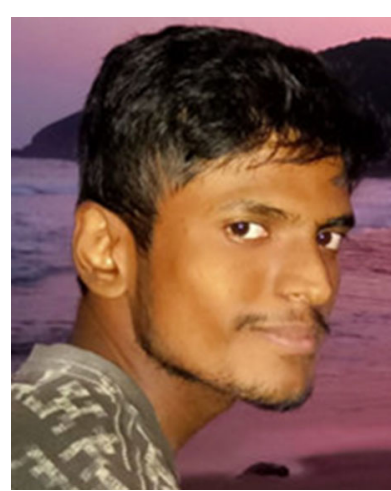

Paidi Dinesh is a Graduated scholar in the Department of Computer Science and Engineering, Sri Sivani College of Engineering, Srikakulam, AP, India. His research interest includesmachine learning and soft computing.

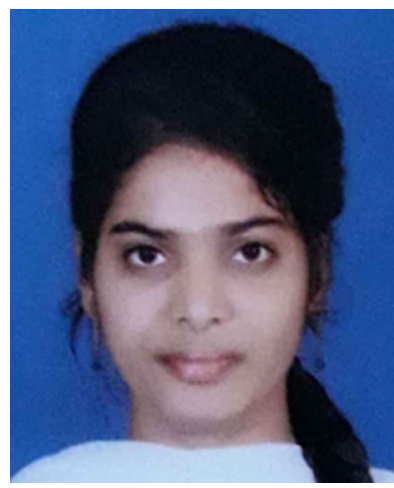

Kanithi Vakula is a Graduated scholar in the Department of Co m puter S c i e c e and Engineering, Sri Sivani College of Engineering, Srikakulam, AP, India. Her research interestincludes data mining, optimisation algorithms, machine learning.

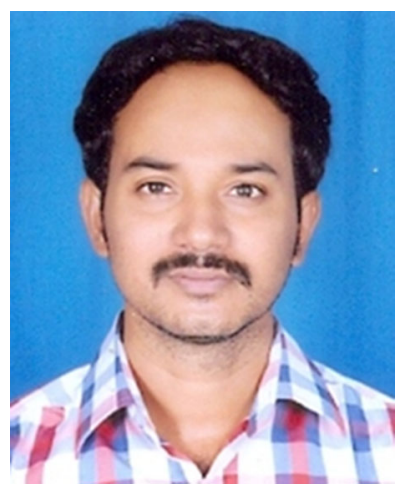

B. Kameswara Rao working as Associate Professor in Aditya Institute of Technology and Management(AITAM), Tekkali, Andhra Pradesh, India. $\mathrm{He}$ is having more than 10 years of teachingexperience. He has published good number of research articles in reputed I n t e r n a t i o n a $1 \quad \mathrm{P}$ e e r reviewed journals and conferences. His research area of interest is Mobile Adhoc Networks, Machine Learning, IoT, and cloud computing. 